\title{
Continuous Record Laplace-based Inference about the Break Date in Structural Change Models*
}

\author{
Alessandro CAsini ${ }^{\dagger}$ \\ University of Rome Tor Vergata \\ PIERRE PERRON ${ }^{\ddagger}$ \\ Boston University
}

13th May 2020

\begin{abstract}
Building upon the continuous record asymptotic framework recently introduced by Casini and Perron (2020a) for inference in structural change models, we propose a Laplace-based (Quasi-Bayes) procedure for the construction of the estimate and confidence set for the date of a structural change. It is defined by an integration rather than an optimization-based method. A transformation of the least-squares criterion function is evaluated in order to derive a proper distribution, referred to as the Quasi-posterior. For a given choice of a loss function, the Laplace-type estimator is the minimizer of the expected risk with the expectation taken under the Quasi-posterior. Besides providing an alternative estimate that is more precise-lower mean absolute error (MAE) and lower root-mean squared error (RMSE) - than the usual least-squares one, the Quasi-posterior distribution can be used to construct asymptotically valid inference using the concept of Highest Density Region. The resulting Laplace-based inferential procedure is shown to have lower MAE and RMSE, and the confidence sets strike a better balance between empirical coverage rates and average lengths of the confidence sets relative to traditional long-span methods, whether the break size is small or large.
\end{abstract}

JEL Classification: C12, C13, C22

Keywords: Asymptotic distribution, bias, break date, change-point, Generalized Laplace, infill asymptotics, semimartingale

${ }^{*}$ We are grateful to the Guest Editor, Timothy Vogelsang, for useful detailed comments. We also thank Zhongjun $\mathrm{Qu}$ and Viktor Todorov for helpful discussions.

${ }^{\dagger}$ Corresponding author at: Department of Economics and Finance, University of Rome Tor Vergata, Via Columbia 2, Rome, 00133, IT. Email: alessandro.casini@uniroma2.it.

${ }^{\ddagger}$ Department of Economics, Boston University, 270 Bay State Road, Boston, MA 02215, US. Email: perron@bu.edu. 


\section{Introduction}

In recent work Casini and Perron (2020a), henceforth CP, developed a continuous record asymptotic framework for inference about the break date in a linear time series regression model with a single shift in some regression parameters while Casini and Perron (2020b) introduced the Generalized Laplace (GL) estimation and inference as an alternative to least-squares in structural change models. In this paper we use the GL method under the continuous record asymptotic framework of CP to tackle some of the problems in this literature. To illustrate the issues involved, consider the simple model $Y_{k h}=\left(\delta_{1}^{0}+\delta^{0} \mathbf{1}_{\left\{k>T_{b}^{0}\right\}}\right) Z_{k h}+e_{k h}$. We have access to $T+1$ observations $(k=0, \ldots, T)$ at equidistant time intervals $h$ over a fixed time span $N=T h$. The parameter $\delta^{0}$ is referred to as the magnitude of the break. The statistical problem is to 1 ) estimate the break date $T_{b}^{0}$ with highest precision possible and, 2) construct confidence sets for this quantity that possess correct empirical coverage rates and short average lengths in finite samples. So far with respect to 1) little work has been done to consider alternatives to OLS in the context of the linear model. With respect to 2) several methods have been investigated, some of which work well with small breaks, while others work well with large ones, none of which deliver adequate coverage rates and "decent" lengths of the confidence sets over all break sizes. We refer to Casini and Perron (2019), Chang and Perron (2018) and the discussion later for an investigation on the drawbacks of popular existing methods, such as those proposed in Bai (1997) and Elliott and Müller (2007). In contrast, CP developed an alternative asymptotic framework based on a continuous record of observations over a fixed time horizon $[0, N]$. This involves letting the sample size $T$ grow to infinity by requiring the sampling $h \downarrow 0$ at the same rate so that $N=T h$ remains fixed. The limiting distribution depends on structural parameters but feasible inference can be developed.

We consider the asymptotics with $h \downarrow 0$ as an alternative asymptotic experiment that can deliver better approximations for discrete-time applications and not necessarily as a framework that is useful only for high-frequency data. Figures 1-2 present plots of the density of the distribution of the least-squares estimate of the break date for the simple model discussed above with a parameter shift associated to a regressor $Z_{k h}$ specified to follow an $\operatorname{ARMA}(1,1)$ process and i.i.d. Gaussian disturbances $e_{k h}$. The distributions presented are: the exact finite-sample distribution, Bai's (1997) classical large- $N$ limit distribution, $\mathrm{CP}$ 's continuous record limit distribution and its feasible version (which uses plug-in estimates for the true parameter values). The continuous record limiting distributions provide an impressively accurate approximation to the finite-sample distribution for both small (cf. Figure 1) and large break sizes (cf. Figure 2) and does so for different break locations (at one-fourth and half-sample in, respectively, the left and right plots). Additionally, it is evident that the classical shrinkage large- $N$ asymptotic distribution does not offer an accurate approximation, especially when the break magnitude is small (cf. Figure 1).

Bai's (1997) method for the construction of the confidence intervals for $T_{b}^{0}$ relies on standard 
asymptotic arguments with the span increasing such that each regime increases proportionately. This coupled with the standard mixing assumption implies that the limit distribution depends only on a neighborhood around the true value. This is in stark contrast to the finite-sample distribution which, especially for small breaks, is influenced by the location of the break and the properties of the processes over the whole sample. Hence, it should not be surprising that the confidence intervals from Bai's (1997) method display empirical coverage rates often below the nominal level when the break is not large. CP documented the highly non-standard features of the density and consequently proposed an alternative inference procedure based on the concept of Highest Density Region (HDR). Their method was shown to offer the best balance between empirical coverage rates and average lengths of the confidence sets among existing methods.

We develop additional inference procedures for the break date in a linear regression model based on the continuous record asymptotic framework and using the Generalized Laplace estimation method. The aim is to provide both a more precise estimate and methods to construct confidence sets with good finite-sample properties. The idea behind the Laplace (or Quasi-Bayes) estimator goes back to Laplace (1774). For a recent application, see Chernozhukov and Hong (2003) for regular problems oriented toward microeconometric applications [see also Forneron and $\mathrm{Ng}$ (2018) for a review and comparisons]. Laplace-type estimators rely on statistical criterion functions and integration-based estimation rather than optimization. They are constructed as integral transformations of extremum criterion functions and can be computed using Markov Chain Monte Carlo methods (MCMC). The integral transformation of the criterion function is meant to provide an approximation to the likelihood approach. Hence, they are often referred to as Quasi-Bayesian estimators. Since no parametric likelihood is available, such a transformation turns the objective function into a proper density - referred to as the Quasi-posterior - over the parameter of interest. Our goal is to consider Laplace estimators and HDR methods to construct the confidence sets, as alternatives to the least-squares estimator coupled with classical inference procedure, which have better finite sample properties, namely reduced Mean Absolute Error (MAE) and Root-Mean Squared Error (RMSE), and confidence sets with accurate coverage rates and short lengths. Of particular importance, we aim to achieve this goal whatever the size of the break, a notoriously difficult problem in the structural change literature.

The use of Laplace-type estimators in Chernozhukov and Hong (2003) was mainly motivated by a need to circumvent the curse of dimensionality inherent in the computation of some classical extremum estimators. Furthermore, they focused on regular problems for which a quadratic expansion of the criterion function is available. Our implementation stems from different concerns. First, our motivation does not arise from a practical need of reducing the curse of dimensionality inherent to computation. Indeed, the least-squares estimates are simple to compute even in models with multiple changes [cf. Hawkins (1976) and Bai and Perron (2003) for an efficient algorithm based on the principle of dynamic programming]. Using the Laplace-type estimator, we aim to have better 
estimates than those based on the least-squares principle; e.g., reduced MAE and RMSE. This should not come at the expense of less adequate confidence sets. Hence, we use the Laplace-type estimator in conjunction with the continuous record limiting distribution and the HDR method to construct confidence sets. A second crucial distinction is that estimating the date of a structural change is a non-standard statistical problem. The limiting distribution of the break point estimator is related to the location of the maximum of a two-sided Gaussian process with drift and thus quite different from the asymptotic distribution of regular estimators. More importantly, the objective function does not admit a quadratic expansion, which adds technical complexities. Although the definition of the Laplace estimator in our context is similar to Chernozhukov and Hong (2003), its asymptotic properties are different in that the contribution of information provided by the prior function does not vanish asymptotically due to the non-regularities of the structural change problem. Hence, the estimator does not share the same Quasi-Bayesian characterization used by Chernozhukov and Hong (2003) in regular problems. In addition, our estimator also differs from the Expectation-Maximization (EM) algorithm [see Dempster, Laird, and Rubin (1977)] because we compute the expected risk for a given loss function and not just the expected value of some log-likelihood function. However, what our approach shares with the EM algorithm is that when the "Quasi-prior" defined below is used, then our estimator also relies on a two-step procedure.

The Laplace-type estimation is explored as follows. We begin with the least-squares estimation of the break date and present the feasible limit distribution developed by CP under a continuous record framework. We treat the corresponding density function as our "prior" and call it the "Quasi-prior". ${ }^{1}$ We compute a transformation of the least-squares criterion function and combine it with the Quasi-prior in order to derive a proper distribution which we refer to as the "Quasiposterior". We then use simple computational methods based on integration to define the so-called "Generalized Laplace" (GL) estimator. Asymptotically valid inferences are constructed using HDRtype methods. The main idea behind the usefulness of our method can be explained as follows. First, note that the least-squares objective function is quite flat with respect to the candidates break dates $T_{b}$. However, the distribution of the least-squares estimate is quite informative in that it assigns sharply different density mass across $T_{b}$ (c.f. the multi-modal feature). Hence, working with the objective function weighted by a Quasi-prior set equal to the density of the least-square estimate yields estimates with better properties as shown in the simulations. Using a least-absolute deviation loss function, the Laplace estimate with our chosen quasi-prior is shown to have substantially lower MAE and RMSE in finite-samples compared to that obtained using least-squares. As in other methods, our procedure relies on a trimming parameter $0<\epsilon<1$ which prevents the estimator to locate the break date in the first and last $100 \epsilon \%$ of the sample. To

\footnotetext{
${ }^{1}$ This distribution should not be viewed as a prior in Bayesian sense. Indeed, it should be simply interpreted as a weight function. Furthermore, it should be noted that this step implicitly relies on an optimization procedure which may not be present under the setting of Chernozhukov and Hong (2003).
} 
achieve good results it is necessary that the trimming parameter should not be chosen too high because otherwise the estimate might tend to overestimate (resp. underestimate) the break date if it is in the first (resp. second) half of the sample. With regards to the GL estimator, the smaller the trimming the larger is the information used by the procedure. Since its construction relies on the overall behavior of the criterion function we find that $\epsilon=0.05$ performs well for different locations of the break date. Our proposed inference results in confidence sets for the break date with more accurate coverage rates and often accompanied by a non-trivial reduction in the width of the confidence set relative to popular methods.

Recently Baek (2019) proposed to modify the least-squares objective function by applying some weights so as to reduce the multimodality effect. The latter estimator displays lower RMSE but has often higher bias relative to the original LS estimator. The latter may be due to the fact that the weighting is not data-dependent while our approach fully exploits the information from the criterion function since the weighting or quasi-prior is data-dependent.

This paper relates to two recent papers, namely CP (2020a, 2020b). CP developed the continuous record asymptotics that we use in this paper and proposed confidence sets for the break date based on the continuous record asymptotic distribution. Hence, it contains the essential ingredients for our proposed GL method, while at the same time offering alternative inference procedures. Casini and Perron (2020b) analyzed the GL method under classical asymptotics and focused on the theoretical relationship between the asymptotic distribution of frequentist and Bayesian estimators of the break point. They showed that depending on some input parameter, the GL estimator exhibits a dual limit distribution - the shrinkage asymptotic distribution of Bai and Perron (1998), or a Bayes-type asymptotic distribution [cf. Ibragimov and Has'minskiǐ (1981)]. Moreover, the results in the former extend to models with multiple breaks and models with trending regressors which are not covered in this paper. Finally, some of the results in this paper allow for long-memory which is ruled out by Casini and Perron (2020b). See also Phillips (1987) and Perron (1991) for early applications of continuous record asymptotics in the context of unit roots.

Recent works have used continuous-time asymptotics for structural change and nonstationarity models more generally. Besides the already discussed approach of CP, Jiang, Wang, and $\mathrm{Yu}$ (2018) studied the finite-sample bias of a break point estimator for a univariate diffusion with constant volatility and a change-point in the drift while Jiang, Wang, and Yu (2017) focused on the break point estimator for the Ornstein-Uhlenbeck process. Their approach is different from CP in that their results are less general and feasible inference for the break point is not discussed. Finally, Chambers and Taylor (2019) considered both deterministic one-time and continuous stochastic parameter change in a continuous-time autoregressive model while Casini (2018) introduced continuous-time asymptotics to test for forecast failure.

The paper is organized as follows. Section 2 introduces the setup. Section 3 summarizes results from CP needed for subsequent analyses. In Section 4, we develop the large-sample properties of 
the GL estimators. We verify their accuracy in Section 5. Section 6 describes the inference methods proposed, which are evaluated via simulations Section 7. Section 8 offers brief concluding remarks. All technical derivations are contained in a supplement [Casini and Perron (2020c)].

\section{The Statistical Setting}

Section 2.1 introduces the model and some assumptions. We also provide a discussion of our assumptions and the relationship of our framework with that of the long-span (shrinkage) asymptotics considered by Bai (1997), Bai and Perron (1998) and Perron and Qu (2006). The least-squares estimator is defined in Section 2.3. The following notations are used in the sequel. All limits are taken as $T \rightarrow \infty$ with the span $N$ kept fixed, where $N=T h$ with $h$, the sampling interval so that $h \downarrow 0$ at the same rate as $T \rightarrow \infty$. $\mathbb{R}$ denotes the set of real numbers. For two vectors $a$ and $b$, we write $a \leq b$ if the inequality holds component-wise. We denote the transpose of a matrix $A$ by $A^{\prime}$. The $(i, j)$ elements of $A$ are denoted by $A^{(i, j)}$. We use $\|\cdot\|$ to denote the Euclidean norm of a linear space i.e., $\|x\|=\left(\sum_{i=1}^{p} x_{i}^{2}\right)^{1 / 2}$ for $x \in \mathbb{R}^{p}$. For a matrix $A$ we use the vector-induced norm, i.e., $\|A\|=\sup _{x \neq 0}\|A x\| /\|x\|$. For a sequence of matrices $\left\{A_{T}\right\}$, we write $A_{T}=o_{\mathbb{P}}(1)$ if each of its elements is $o_{\mathbb{P}}(1)$ and likewise for $O_{\mathbb{P}}(1)$. The symbol $\lfloor\cdot\rfloor$ denotes the largest smaller integer function while $\otimes$ is used for the product of $\sigma$-fields. A sequence $\left\{u_{k h}\right\}_{k=1}^{T}$ is i.i.d. (resp., i.n.d.) if the $u_{k h}$ are independent and identically (resp., non-identically) distributed. We use $\stackrel{\mathbb{P}}{\rightarrow}$, and $\Rightarrow$ to denote, respectively, convergence in probability (under some measure $\mathbb{P}$ ), and weak convergence, while $\stackrel{\mathcal{L}_{-} \text {s }}{\Rightarrow}$ is used to denote stable convergence in law. We write $\mathscr{M} \mathscr{N}(a, b)$ for the mixed normal distribution with parameters $(a, b)$. The space $\mathbb{M}_{p}^{\text {càdlàg }}$ collects all $p \times p$ positive definite real-valued matrices whose elements are càdlàg. We use the superscript + in $\mathbb{M}_{p}$ if these matrices are required to be positive definite. $\boldsymbol{F} \boldsymbol{V}^{\mathrm{c}}$ denotes the class of continuous adapted finite variation processes and $\boldsymbol{M}_{\mathrm{loc}}^{\mathrm{c}}$ denotes the class of continuous local martingale processes with finite positive definite conditional variance. For semimartingales $\left\{S_{t}\right\}_{t \geq 0}$ and $\left\{V_{t}\right\}_{t \geq 0}$, we denote their covariation process by $[S, V]_{t}$ and its predictable counterpart by $\langle S, V\rangle_{t}$. We anticipate that in our setting the latter two processes will be equivalent. The symbol "ڤ” refers to definitional equivalence.

\subsection{The Model}

A standard discrete-time partial structural change model with a single break is given by:

$$
\begin{array}{ll}
Y_{t}=D_{t}^{\prime} \varrho^{0}+Z_{t}^{\prime} \delta_{1}^{0}+u_{t}, & \left(t=0, \ldots, T_{b}^{0}\right) \\
Y_{t}=D_{t}^{\prime} \varrho^{0}+Z_{t}^{\prime} \delta_{2}^{0}+u_{t}, & \left(t=T_{b}^{0}+1, \ldots, T\right),
\end{array}
$$


where $Y_{t}$ is the dependent variable, $D_{t}$ and $Z_{t}$ are, respectively, $p \times 1$ and $q \times 1$ vectors of regressors and $e_{t}$ is an unobservable disturbance. The statistical problem is to estimate the unknown parameters $\varrho^{0}, \delta_{1}^{0}, \delta_{2}^{0}$ and the break date $T_{b}^{0}$. A structural change occurs at $T_{b}^{0}$ because by assumption $\delta_{1}^{0} \neq \delta_{2}^{0}$. The break magnitude is $\delta^{0}=\delta_{2}^{0}-\delta_{1}^{0}$. The inference problem is to construct estimates and confidence sets for the break date $T_{b}^{0}$ when $T+1$ observations on $\left(Y_{t}, D_{t}, Z_{t}\right)$ are available.

We introduce the high-frequency setting which serves as a probabilistic background on which our asymptotic arguments are developed. There is a filtered probability space $\left(\Omega, \mathscr{F},\left(\mathscr{F}_{t}\right)_{t \geq 0}, \mathbb{P}\right)$ on which the continuous time process $X \triangleq\left(Y, D^{\prime}, Z^{\prime}\right)^{\prime}$ is defined, where $Y \triangleq\left\{Y_{t}\right\}_{t \geq 0}, D \triangleq$ $\left\{D_{t}\right\}_{t \geq 0}, Z \triangleq\left\{Z_{t}\right\}_{t \geq 0}$ are assumed to be Itô semimartingales. We observe $T+1$ realizations of $Y_{t}, D_{t}$ and $Z_{t}$ at equidistant discrete times $t=0, h, 2 h, \ldots, N$, where $N \triangleq T h$ is the length of the fixed time span $[0, N]$. Note that in general $N$ is not identified and could be normalized to one. However, we keep a generic $N$ throughout to allow a better intuitive understanding of the results. Under the continuous record asymptotic scheme, we let the sample size $T$ grow by shrinking the sampling interval $h$ to zero so that the span $N$ remains fixed.

For each $h, D_{k h} \in \mathbb{R}^{p}$ and $Z_{k h} \in \mathbb{R}^{q}$ are random vector step functions changing at times $0, h, 2 h, \ldots, T h$. The continuous-time unobservable error sequence is the càdlàg adapted process $\left\{e_{t}^{*}\right\}$. The regressors $D_{k h}$ and $Z_{k h}$ include locally-integrable semimartingales (extended to allow for predictable processes in Section 2.2). We assume that the discretized processes $D_{k h}$ and $Z_{k h}$ are adapted to the increasing and right-continuous filtration $\left\{\mathscr{F}_{t}\right\}$. By the Doob-Meyer decomposition we can write, ${ }^{2}$ for $k=1, \ldots, T: \Delta_{h} D_{k} \triangleq \mu_{D, k} h+\Delta_{h} M_{D, k}$ and $\Delta_{h} Z_{k} \triangleq \mu_{Z, k} h+\Delta_{h} M_{Z, k}$, where the drifts $\mu_{D, t} \in \mathbb{R}^{p}, \mu_{Z, t} \in \mathbb{R}^{q}$ are $\mathscr{F}_{t-h}$-measurable, and $M_{D, k} \in \mathbb{R}^{p}, M_{Z, k} \in \mathbb{R}^{q}$ are continuous local martingales with $\mathbb{P}$-a.s. finite conditional covariance matrices $\mathbb{E}\left(\Delta_{h} M_{D, t} \Delta_{h} M_{D, t}^{\prime} \mid \mathscr{F}_{t-h}\right)=$ $\Sigma_{D, t-h} \Delta t$ and $\mathbb{E}\left(\Delta_{h} M_{Z, t} \Delta_{h} M_{Z, t}^{\prime} \mid \mathscr{F}_{t-h}\right)=\Sigma_{Z, t-h} \Delta t$. Exact assumptions will be given below. To the continuous-time process $\left\{e_{t}^{*}\right\}$ corresponds a disturbance sequence $\left\{\Delta_{h} e_{t}^{*}\right\}$ such that $\left\{\Delta_{h} e_{t}^{*}, \mathscr{F}_{t}\right\}$ is a continuous local martingale difference sequence with finite conditional variance given by $\mathbb{E}\left[\left(\Delta_{h} e_{t}^{*}\right)^{2} \mid \mathscr{F}_{t-h}\right]=\sigma_{e, t-h}^{2} \Delta t \mathbb{P}$-a.s. Under this formulation, the discretized model is

$$
\Delta_{h} Y_{k} \triangleq \begin{cases}\left(\Delta_{h} D_{k}\right)^{\prime} \varrho^{0}+\left(\Delta_{h} Z_{k}\right)^{\prime} \delta_{1}^{0}+\Delta_{h} e_{k}^{*}, & \left(k=1, \ldots,\left\lfloor T \lambda_{0}\right\rfloor\right) \\ \left(\Delta_{h} D_{k}\right)^{\prime} \varrho^{0}+\left(\Delta_{h} Z_{k}\right)^{\prime} \delta_{2}^{0}+\Delta_{h} e_{k}^{*}, & \left(k=\left\lfloor T \lambda_{0}\right\rfloor+1, \ldots, T\right)\end{cases}
$$

where $\lambda_{0} \in(0,1), \varrho^{0} \in \mathbb{R}^{p}, \delta_{1}^{0}, \delta_{2}^{0} \in \mathbb{R}^{q}$, and $\delta^{0} \triangleq \delta_{2}^{0}-\delta_{1}^{0}$. It holds that $\delta^{0} \neq 0$ so that a structural change in the parameter associated with $\Delta_{h} Z_{k}$ has occurred at time $\left\lfloor N \lambda_{0}\right\rfloor$. Under our setting, we need to distinguish between the actual break date $N_{b}^{0}=N \lambda_{0}$ (on a calendar time) and the index of the discrete-time observation associated with the break point: $T_{b}^{0} \triangleq\left\lfloor T \lambda_{0}\right\rfloor=\left\lfloor N_{b}^{0} / h\right\rfloor$. Under the classical long-span setting this is not necessary since $h=1$ or $N=T$.

\footnotetext{
${ }^{2}$ For any process $X$ we denote the "increments" of $X$ by $\Delta_{h} X_{k}=X_{k h}-X_{(k-1) h}$. We sometimes use $\Delta t$ in place of $h$ in order to conveniently make our sums look like integrals.
} 
The specification implicitly assumes the following continuous-time data-generating process,

$$
D_{t}=D_{0}+\int_{0}^{t} \mu_{D, s} d s+\int_{0}^{t} \sigma_{D, s} d W_{D, s}, \quad Z_{t}=Z_{0}+\int_{0}^{t} \mu_{Z, s} d s+\int_{0}^{t} \sigma_{Z, s} d W_{Z, s}
$$

where $\mu_{D, t}$ (resp., $\mu_{Z, t}$ ) is the infinitesimal conditional mean of $D_{t}$ (resp., $Z_{t}$ ) and takes value in $\mathbb{R}^{p}$ (resp., $\left.\mathbb{R}^{q}\right) ; \sigma_{D, t}$ and $\sigma_{Z, t}$ are the instantaneous covariance processes taking values in $\mathbb{M}_{p}^{\text {càdlàg }}$ and $\mathbb{M}_{q}^{\text {càdlàg }}$, respectively; $W_{D}$ (resp., $W_{Z}$ ) is a $p$ (resp., $q$ )-dimensional standard Wiener process; and $D_{0}$ and $Z_{0}$ are $\mathscr{F}_{0}$-measurable random vectors. The process $e_{t}^{*}$ is a continuous local martingale orthogonal (in martingale sense) to both $D_{t}$ and $Z_{t}$, i.e., $\langle e, D\rangle_{t}=\langle e, Z\rangle_{t}=0$ for all $t$. We consider only processes with continuous sample paths, as stated in the following assumption.

Assumption 2.1. D, Z, e and $\Sigma^{0} \triangleq\left\{\Sigma_{\cdot, t}, \sigma_{e, t}\right\}_{t \geq 0}$ are continuous Itô semimartingales.

Assumption 2.1 rules out processes with discontinuous sample paths from our analysis. Hence, our results are not expected to provide good approximations for applications involving highfrequency data for which jumps are likely to be important; e.g., Andersen, Fusari, and Todorov (2016), Bandi and Renò (2016) and Hounyo, Liu, and Varneskov (2020) for financial-oriented applications and Li and Xiu (2016) for a GMM setup; for a textbook account, see Aït-Sahalia and Jacod (2014). Another important difference from the high-frequency statistics centers on the "mean effect" which is captured here by the drift process. This poses several challenges because the drift is not identified under a continuous record asymptotics. CP dealt with this issue by introducing a so-called small-dispersion model, for which the parameters affecting the limit distribution can be consistently estimated and all theoretical results are shown to apply to this case as well (c.f. Section 2.2 below).

Assumption 2.2. The model (2.2)-(2.3) satisfies the following: (i) the càdlàg processes $\sigma_{D, t}$ and $\sigma_{Z, t}$ are locally bounded; (ii) $\mu_{D, t}$ and $\mu_{Z, t}$ are locally bounded. $\int_{0}^{t} \mu_{D, s} d s$ and $\int_{0}^{t} \mu_{Z, s} d s$ belong to $\boldsymbol{F} \boldsymbol{V}^{\mathrm{c}}$; (iii) $\int_{0}^{t} \sigma_{D, s} d W_{D, s}, \int_{0}^{t} \sigma_{Z, s} d W_{Z, s} \in \boldsymbol{M}_{\mathrm{loc}}^{\mathrm{c}}$. Further, they possess $\mathbb{P}$-a.s. finite positive definite conditional variances defined by $\Sigma_{D, t}=\sigma_{D, t} \sigma_{D, t}^{\prime}$ and $\Sigma_{Z, t}=\sigma_{Z, t} \sigma_{Z, t}^{\prime}$, which for all $t<\infty$ satisfy, for $j=1, \ldots, p: \int_{0}^{t} \Sigma_{D, s}^{(j, j)} d s<\infty$ and $\int_{0}^{t} \Sigma_{Z, s}^{(j, j)} d s<\infty$ where $\Sigma_{D, t}^{(j, r)}$ denotes the $(j, r)$-th element of the process $\Sigma_{D, t}$. Furthermore, for every $j=1, \ldots, p, r=1, \ldots, q$, and $k=1, \ldots, T$, $h^{-1} \int_{(k-1) h}^{k h} \Sigma_{D, s}^{(j, j)} d s$ and $h^{-1} \int_{(k-1) h}^{k h} \Sigma_{Z, s}^{(r, r)} d s$ are finite and bounded away from zero, uniformly in $k$ and $h ;(i v) e^{*} \triangleq\left\{e_{t}^{*}\right\}_{t \geq 0}$ is a continuous local martingale satisfying $e_{t}^{*} \triangleq \int_{0}^{t} \sigma_{e, s} d W_{e, s}$ with $0<\sigma_{e, t}^{2}<\infty$ for all $t \geq 0$, where $W_{e}$ is a Wiener process. Also, $\langle e, D\rangle_{t}=\langle e, Z\rangle_{t}=0, t \geq 0$.

Parts (i)-(iii) contains regularity conditions imposed in the high-frequency financial statistics literature [cf. Barndorff-Nielsen and Shephard (2004), Li, Todorov, and Tauchen (2017) and Li and Xiu (2016)]. Part (iv) specifies the disturbance process to possess continuous sample paths and to be contemporaneously orthogonal to the regressors. Assumptions 2.1-2.2 imply that the 
processes in our model are diffusion-type processes if one further assumes that the volatilities are deterministic. We shall not impose the latter condition. As a consequence, $X=\left(Y, D^{\prime}, Z^{\prime}\right)^{\prime}$ is a member of the continuous stochastic volatility semimartingale class. We model volatility as a latent factor since it does not pose any substantial impediment for the development of our theoretical results and they are more general including, for example, nonstationarity and long-memory.

Assumption 2.3. $N_{b}^{0}=\left\lfloor N \lambda_{0}\right\rfloor$, for some $\lambda_{0} \in(0,1)$.

Assumption 2.3 dictates the asymptotic framework adopted and implies that the break date occurs at the observation-index $T_{b}^{0}=T \lambda_{0}$, where $T_{b}^{0}=N_{b}^{0} / h$. Under our framework it implies that the pre- and post-break segments of the sample remain fixed. The usual assumption under the classical large- $N$ asymptotics implies that the time horizons before and after the break date grow proportionately, which, along with the mixing assumption implies that only a small neighborhood around the true break date is relevant asymptotically, thereby ruling out the possibility to discern any asymmetric feature of the asymptotic distribution simply caused by the location of the break date. The continuous record asymptotic framework preserves information about the data span and the location of the break, and the mixing and ergodic assumptions are not needed. CP showed that the theoretical results derived for conducting inference about the break date in model (2.2) are applicable to classical structural change models for which a long-span setting is usually adopted. It is convenient to use the following re-parametrization. Let $y_{k h}=\Delta_{h} Y_{k h}, x_{k h}=\left(\Delta_{h} D_{k}^{\prime}, \Delta_{h} Z_{k}^{\prime}\right)^{\prime}$, $z_{k h}=\Delta_{h} Z_{k}, e_{k h}=\Delta_{h} e_{k}^{*}$, and $\beta^{0}=\left(\left(\varrho^{0}\right)^{\prime},\left(\delta_{1}^{0}\right)^{\prime}\right)^{\prime}$. Then, (2.2) is

$$
\begin{array}{lr}
y_{k h}=x_{k h}^{\prime} \beta^{0}+e_{k h}, & \left(k=1, \ldots, T_{b}^{0}\right) \\
y_{k h}=x_{k h}^{\prime} \beta^{0}+z_{k h}^{\prime} \delta^{0}+e_{k h}, & \left(k=T_{b}^{0}+1, \ldots, T\right) .
\end{array}
$$

We further define the full column rank $(p+q) \times q$ known matrix $H$ such that $z_{k h}=H^{\prime} x_{k h}$. We can then state model (2.4) in matrix format, which will be used for the derivations. Let $Y=\left(y_{h}, \ldots, y_{T h}\right)^{\prime}, X=\left(x_{h}, \ldots, x_{T h}\right)^{\prime}, e=\left(e_{h}, \ldots, e_{T h}\right)^{\prime}, X_{1}=\left(x_{h}, \ldots, x_{T_{b} h}, 0, \ldots, 0\right)^{\prime}, X_{2}=$ $\left(0, \ldots, 0, x_{\left(T_{b}+1\right) h}, \ldots, x_{T h}\right)^{\prime}$ and $X_{0}=\left(0, \ldots, 0, x_{\left(T_{b}^{0}+1\right) h}, \ldots, x_{T h}\right)^{\prime}$. Also, $Z_{1}=X_{1} H, Z_{2}=$ $X_{2} H$ and $Z_{0}=X H$. Then (2.4) is equivalent to

$$
Y=X \beta^{0}+Z_{0} \delta^{0}+e
$$

\subsection{The Extended Model with Predictable Processes}

The assumptions on $D_{t}$ and $Z_{t}$ specify that these processes are continuous Itô semimartingales of the form (2.3). This precludes predictable processes, which are often of interest in applications; e.g., a constant and/or a lagged dependent variable. Technically, these require a separate treatment 
since the coefficients associated with predictable processes are not identified under a fixed-span asymptotic setting. CP considered the following extended model:

$$
\Delta_{h} Y_{k} \triangleq \begin{cases}\mu_{1, h} h+\alpha_{1, h} Y_{(k-1) h}+\left(\Delta_{h} D_{k}\right)^{\prime} \varrho^{0}+\left(\Delta_{h} Z_{k}\right)^{\prime} \delta_{Z, 1}^{0}+\Delta_{h} e_{k}^{*}, & \left(k=1, \ldots,\left\lfloor T \lambda_{0}\right\rfloor\right) \\ \mu_{2, h} h+\alpha_{2, h} Y_{(k-1) h}+\left(\Delta_{h} D_{k}\right)^{\prime} \varrho^{0}+\left(\Delta_{h} Z_{k}\right)^{\prime} \delta_{Z, 2}^{0}+\Delta_{h} e_{k}^{*}, & \left(k=\left\lfloor T \lambda_{0}\right\rfloor+1, \ldots, T\right)\end{cases}
$$

for some given initial value $Y_{0}$. We specify the parameters associated with the constant and the lagged dependent variable as increasing as $h \downarrow 0$ in order for some fixed true parameter to remain in the asymptotics. This is done by specifying: $\mu_{1, h} \triangleq \mu_{1}^{0} h^{-1 / 2}, \mu_{2, h} \triangleq \mu_{2}^{0} h^{-1 / 2}, \mu_{\delta, h} \triangleq \mu_{2, h}-\mu_{1, h}$, $\alpha_{1, h} \triangleq \alpha_{1}^{0} h^{-1 / 2}, \alpha_{2, h} \triangleq \alpha_{2}^{0} h^{-1 / 2}$ and $\alpha_{\delta, h} \triangleq \alpha_{2, h}-\alpha_{1, h}$. Our framework is then similar to the smalldiffusion setting which has been extensively studied in the statistics literature [cf. Ibragimov and Has'minskiǐ (1981), Laredo (1990) and Sorensen and Uchida (2003)]. The results to be discussed below go through with modifications using the results in CP. In particular, a two-step procedure is needed to estimate the parameters as discussed in Section A.2 in CP. The model and results can be trivially extended to allow more general forms of predictable processes.

\subsection{The Least-Squares Estimator of the Break Date}

CP considered the break date least-squares (LS) estimator $\widehat{N}_{b}^{\mathrm{LS}}=\widehat{T}_{b}^{\mathrm{LS}} h$ defined as the minimizer of the sum of squares residuals $S_{h}\left(\theta, N_{b}\right)$ from $(2.4)$, where $\theta \triangleq\left(\beta^{\prime}, \delta^{\prime}\right)^{\prime}$. The parameter vector $\theta$ can be concentrated out of the criterion function to yield an optimization problem over $N_{b}$ only:

$$
\widehat{\theta}_{h}^{\mathrm{LS}}\left(N_{b}\right)=\underset{\theta}{\operatorname{argmin}} S_{h}\left(\theta, N_{b}\right), \quad \widehat{N}_{b}^{\mathrm{LS}}=\underset{h q \leq N_{b} \leq N}{\operatorname{argmin}} S_{h}\left(\widehat{\theta}^{\mathrm{LS}}\left(N_{b}\right),\left(N_{b}\right)\right)
$$

Using a correspondence between the sum of squared residuals and the sup-Wald statistic, we have:

$$
\underset{h q \leq N_{b} \leq N}{\operatorname{argmin}} S_{h}\left(\widehat{\theta}^{\mathrm{LS}}\left(N_{b}\right), N_{b}\right)=\underset{h q \leq N_{b} \leq N}{\operatorname{argmax}} \widehat{\delta}^{\mathrm{LS}}\left(N_{b}\right)^{\prime}\left(Z_{2}^{\prime} M_{X} Z_{2}\right) \widehat{\delta}^{\mathrm{LS}}\left(N_{b}\right)
$$

where $M_{X} \triangleq I-X\left(X^{\prime} X\right) X^{\prime}$ and $\widehat{\delta}^{\mathrm{LS}}\left(N_{b}\right)$ is the LS estimator of $\delta^{0}$ obtained by regressing $Y$ on $X$ and $Z_{2}$. This follows since $Q_{h}\left(\widehat{\theta}^{\mathrm{LS}}\left(N_{b}\right), N_{b}\right) \triangleq \widehat{\delta}^{\mathrm{LS}}\left(N_{b}\right)^{\prime}\left(Z_{2}^{\prime} M_{X} Z_{2}\right) \widehat{\delta}^{\mathrm{LS}}\left(N_{b}\right)$ is the numerator of a modified sup-Wald statistic. The GL estimator will depend on the criterion function $Q_{h}\left(\theta\left(N_{b}\right), N_{b}\right)$ evaluated at each possible break date. Hence, we need the following assumptions.

Assumption 2.4. $\theta^{0}=\left(\left(\beta^{0}\right)^{\prime},\left(\delta^{0}\right)^{\prime}\right)^{\prime} \in \Theta \subset \mathbb{R}^{\operatorname{dim}(\theta)}$, a compact parameter space.

Assumption 2.5. There exists an $l_{0}$ such that for all $l>l_{0}$, the matrices $(l h)^{-1} \sum_{k=1}^{l} x_{k h} x_{k h}^{\prime}$, $(l h)^{-1} \sum_{k=T-l+1}^{T} x_{k h} x_{k h}^{\prime},(l h)^{-1} \sum_{k=T_{b}^{0}-l+1}^{T_{0}^{0}} x_{k h} x_{k h}^{\prime}$, and $(l h)^{-1} \sum_{k=T_{b}^{0}+1}^{T_{b}^{0}+l} x_{k h} x_{k h}^{\prime}$ have minimum eigenvalues bounded away from zero in probability. 
Assumption 2.6. Let $\bar{Q}_{h}\left(\theta^{0}, N_{b}\right) \triangleq \mathbb{E}\left[Q_{h}\left(\theta^{0}, N_{b}\right)-Q_{h}\left(\theta^{0}, N_{b}^{0}\right)\right]$. There exists a $N_{b}^{0}$ such that $\bar{Q}_{h}\left(\theta^{0}, N_{b}^{0}\right)>\sup _{\left(\theta^{0}, N_{b}\right) \notin \mathbf{B}} \bar{Q}_{h}\left(\theta^{0}, N_{b}\right)$, for every open set $\mathbf{B}$ that contains $\left(\theta^{0}, N_{b}^{0}\right)$.

Assumption 2.5 is in the same spirit as A2 in Bai and Perron (1998). It requires that there be enough variation around the break point so that it can be identified. Multiplying the increment $x_{k h}$ by the factor $h^{-1 / 2}$ allows one to normalize $x_{k h}$ so that the assumption is implied by a weak law of large numbers. Assumption 2.6 is a conventional uniqueness condition.

\section{Asymptotic Results for Least-squares Estimation}

In this section, we review continuous record asymptotic results about the break date LS estimator from CP. They provide intuition on the forthcoming asymptotic results about the Laplace estimator since both have a common LS criterion function. The typical asymptotic framework for structural change problems relies on a shrinking shifts assumption. Under a continuous record, we also require a small shifts assumption to analyze the limit distribution of the Laplace estimator. We compare the asymptotics of CP with that of Bai (1997) and Elliott and Müller (2007) in Section 3.2.

\subsection{Continuous Record Asymptotics}

Assumption 3.1. Let $\delta_{h}=h^{1 / 4} \delta^{0}$ and assume that for all $t \in\left(N_{b}^{0}-\epsilon, N_{b}^{0}+\epsilon\right)$, with $\epsilon \downarrow 0$ and $T^{1-\kappa} \epsilon \rightarrow B<\infty, 0<\kappa<1 / 2, \mathbb{E}\left[\left(\Delta_{h} e_{t}^{*}\right)^{2} \mid \mathscr{F}_{t-h}\right]=\sigma_{h, t-h}^{2} \Delta t \mathbb{P}$-a.s, where $\sigma_{h, t} \triangleq \sigma_{h} \sigma_{e, t}$, $\sigma_{h} \triangleq h^{-1 / 4} \bar{\sigma}$ and $\bar{\sigma} \triangleq \int_{0}^{N} \sigma_{e, s}^{2} d s$.

The first part states that the shift parameter converges to zero at a controlled rate. The second allows for a higher degree of uncertainty around the change-point by requiring $\left\{\Delta_{h} e_{t}^{*}\right\}$ to oscillate more as $h \downarrow 0$. The latter neither prevents nor facilitates the identification of the break, i.e., it plays no role for the global properties of the estimator, namely the consistency of $\widehat{N}_{b}$ and $\widehat{\theta}$ as well as the asymptotic distribution of $\widehat{\theta}$, though not that of $\widehat{N}_{b}$.

Under Assumption 3.1, the rate of convergence of the LS estimator is $T^{1-\kappa}$, with $0<\kappa<$ $1 / 2$. This rate is fast and therefore the volatility of the errors is scaled up around the changepoint so that the objective function behaves as if it were a standard diffusion process. Note that the $T^{-(1-\kappa)}$-neighborhood in which the errors have higher variance arises from the $T^{1-\kappa}$-rate of convergence of $\hat{\lambda}_{b}$. Note also that the rate of convergence $T^{1-\kappa}$ is sufficiently fast to guarantee a $\sqrt{T}$ consistent estimation of the slope parameters. The results proved in CP are stated in the following propositions. Let $\Sigma^{*} \triangleq\left\{\mu, t, \Sigma_{\cdot, t}, \sigma_{e, t}\right\}_{t \geq 0}$, and $Z_{\Delta} \triangleq\left(0, \ldots, 0, z_{\left(T_{b}+1\right) h}, \ldots, z_{T_{b}^{0} h}, 0, \ldots, 0\right)$ if 
$T_{b}<T_{b}^{0}$ and $Z_{\Delta} \triangleq\left(0, \ldots, 0, z_{\left(T_{b}^{0}+1\right) h}, \ldots, z_{T_{b} h}, 0, \ldots, 0\right)$ if $T_{b}>T_{b}^{0}$. Define

$$
\Delta_{h} \widetilde{e}_{t} \triangleq\left\{\begin{array}{ll}
\Delta_{h} e_{t}^{*}, & t \notin\left(N_{b}^{0}-\epsilon, N_{b}^{0}+\epsilon\right) \\
h^{1 / 4} \Delta_{h} e_{t}^{*}, & t \in\left(N_{b}^{0}-\epsilon, N_{b}^{0}+\epsilon\right)
\end{array} .\right.
$$

Proposition 3.1. Under Assumptions 2.2-2.6 and 3.1: (i) $\widehat{N}_{b} \stackrel{\mathbb{P}}{\rightarrow} N_{b}^{0}$; (ii) for every $\varepsilon>0$ there exists a $K>0$ such that for all large $T, \mathbb{P}\left(T^{1-\kappa}\left|\widehat{N}_{b}-N_{b}^{0}\right|>K\left\|\delta^{0}\right\|^{-2} \bar{\sigma}^{2}\right)<\varepsilon$; and (iii) for $\kappa \in(0,1 / 4]$, as $T \rightarrow \infty,\left(\sqrt{T / N}\left(\widehat{\beta}-\beta^{0}\right), \sqrt{T / N}\left(\widehat{\delta}-\delta_{h}\right)\right)^{\prime}=O_{\mathbb{P}}(1)$.

The derivation of the continuous record asymptotic distribution uses a change of time scale $s \mapsto \psi_{h}^{-1} t$ where $\psi_{h}=h^{1-\kappa}$. Under fixed-shifts, Proposition 3.2 in CP shows that $\widehat{N}_{b}-N_{b}^{0}=$ $O_{p}\left(T^{-1}\right)$, i.e., $\widehat{N}_{b}$ is in a shrinking neighborhood of $N_{b}^{0}$, which however shrinks too fast and impedes the development of a feasible limit theory. Hence, the need to analyze the objective function in a small neighborhood of the true break date under this "fast time scale". See Section 4.3 for details.

Proposition 3.2. Under Assumptions 2.2-2.6 and 3.1, and under the "fast time scale",

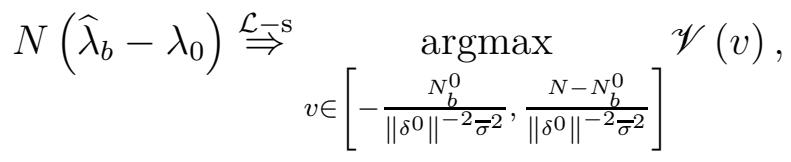

where $\mathscr{V}(v) \triangleq-\left(\delta^{0}\right)^{\prime}\left\langle Z_{\Delta}, Z_{\Delta}\right\rangle(v) \delta^{0}+2\left(\delta^{0}\right)^{\prime} \mathscr{W}(v),\left\langle Z_{\Delta}, Z_{\Delta}\right\rangle(v)$ is the predictable quadratic variation process of $Z_{\Delta}$ and the process $\mathscr{W}(v)$ is, conditionally on the $\sigma$-field $\mathscr{F}$, a two-sided centered Gaussian martingale with independent increments.

To save space, we do not report the explicit expression for the covariance of $\mathscr{W}(v)$; see Theorem 4.1 in CP. Under stationary regimes, Proposition 3.3 presents the corresponding result.

Assumption 3.2. The process $\Sigma^{0}$ is (possibly time-varying) deterministic; $\left\{z_{k h}, e_{k h}\right\}$ is secondorder stationary within each regime. For $k=1, \ldots, T_{b}^{0}, \mathbb{E}\left(z_{k h} z_{k h}^{\prime} \mid \mathscr{F}_{(k-1) h}\right)=\Sigma_{Z, 1} h, \mathbb{E}\left(\widetilde{e}_{k h}^{2} \mid \mathscr{F}_{(k-1) h}\right)=$ $\sigma_{e, 1}^{2} h$ and $\mathbb{E}\left(z_{k h} z_{k h}^{\prime} \widetilde{e}_{k h}^{2} \mid \mathscr{F}_{(k-1) h}\right)=\Omega_{\mathscr{W}, 1} h^{2}$ while for $k=T_{b}^{0}+1, \ldots, T, \mathbb{E}\left(z_{k h} z_{k h}^{\prime} \mid \mathscr{F}_{(k-1) h}\right)=\Sigma_{Z, 2} h$, $\mathbb{E}\left(\widetilde{e}_{k h}^{2} \mid \mathscr{F}_{(k-1) h}\right)=\sigma_{e, 2}^{2} h$ and $\mathbb{E}\left(z_{k h} z_{k h}^{\prime} \widetilde{e}_{k h}^{2} \mid \mathscr{F}_{(k-1) h}\right)=\Omega_{\mathscr{W}, 2} h^{2}$.

Let $W_{i}^{*}(s), i=1,2$, be two independent standard Wiener processes defined on $[0, \infty)$, starting at the origin when $s=0$. Let

$$
\mathscr{V}^{*}(s)= \begin{cases}-\frac{|s|}{2}+W_{1}^{*}(s), & \text { if } s<0 \\ -\frac{\left(\delta^{0}\right)^{\prime} \Sigma_{Z, 2} \delta^{0}}{\left(\delta^{0}\right)^{\prime} \Sigma_{Z, 1} \delta^{0}} \frac{|s|}{2}+\left(\frac{\left(\delta^{0}\right)^{\prime} \Omega_{\mathscr{W}, 2}\left(\delta^{0}\right)}{\left(\delta^{0}\right)^{\prime} \Omega_{\mathscr{W}, 1}\left(\delta^{0}\right)}\right)^{1 / 2} W_{2}^{*}(s), & \text { if } s \geq 0 .\end{cases}
$$


Proposition 3.3. Under Assumptions 2.2-2.6 and 3.1-3.2, and under the "fast time scale",

$$
\frac{\left(\left(\delta^{0}\right)^{\prime} \Sigma_{Z, 1} \delta^{0}\right)^{2}}{\left(\delta^{0}\right)^{\prime} \Omega_{\mathscr{W}, 1} \delta^{0}} N\left(\widehat{\lambda}_{b}-\lambda_{0}\right) \Rightarrow \underset{s \in\left[-\frac{N_{b}^{0}}{\left\|\delta^{0}\right\|^{-2} \bar{\sigma}^{2}} \frac{\left(\left(\delta^{0}\right)^{\prime} \Sigma_{Z, 1} \delta^{0}\right)^{2}}{\left(\delta^{0}\right)^{\prime} \Omega_{\mathscr{W}, 1}\left(\delta^{0}\right)}, \frac{N-N_{b}^{0}}{\left\|\delta^{0}\right\|^{-2} \bar{\sigma}^{2}} \frac{\left(\left(\delta^{0}\right)^{\prime} \Sigma_{Z,, 1^{0}}\right)^{2}}{\left(\delta^{0}\right)^{\prime} \Omega_{\mathscr{W}, 1^{\delta}}}\right]}{\operatorname{argmax}} \mathscr{V}^{*}(s) .
$$

\subsection{Comparison with Other Approaches}

As shown in Figures 1-2, the structural change problem is characterized by a high degree of uncertainty when the break magnitude is not large. The classical shrinkage asymptotics of Bai (1997), with $\delta_{T}$ converging to zero at a rate slower than $O\left(T^{1 / 2}\right)$, underestimates the degree of uncertainty and, as the figures show, it provides a poor approximation to the finite-sample behavior of the LS estimator. CP argued that this issue is also responsible for the poor coverage probabilities of the associated confidence intervals when the break size is small. This asymptotic distribution does not capture important features of the finite-sample distribution such as multimodality, asymmetry and shape changing with the magnitude of $\delta^{0}$. Underestimating the true uncertainty leads to confidence intervals that are too short and consequently undercover.

The goal is to find an asymptotic experiment that delivers a good approximation and leads to inference that is reliable in practice. Elliott and Müller [cf. Elliott and Müller (2007) and Elliott, Müller, and Watson (2015)] proposed an alternative framework where $\delta_{T}=O\left(T^{-1 / 2}\right)$ so that $\delta_{T}$ goes faster to zero. This can be referred to as a weak identification. Although it increases the uncertainty in the problem, the rate at which $\delta_{T}$ goes to zero is too fast in the sense that statistical uncertainty is too high so that $\widehat{\lambda}_{b}=\widehat{T}_{b} / T, \widehat{\delta}_{1}$ and $\widehat{\delta}_{2}$ become inconsistent for $\lambda_{0}, \delta_{1}^{0}$ and $\delta_{2}^{0}$, respectively. This can be problematic and indeed, their inference suffers from the opposite problem in that confidence intervals for $\widehat{T}_{b}$ can be too large and thus uninformative [Casini and Perron (2019), Chang and Perron (2018) and CP]. Furthermore, inconsistency for the regression coefficients is unappealing since researchers often are ultimately interested in making inference about the regression coefficients and not just about $T_{b}^{0}$.

Although the asymptotic experiments considered so far all impose conditions on the break magnitude $\delta^{0}$, CP pointed out that what matters is not just $\delta^{0}$. Consider a location model with a change $\delta^{0}$ in the mean and independent errors. What describe the uncertainty in the model is the ratio $\delta^{0} / \sigma$ where $\sigma$ is the volatility of the errors. Instead of controlling just $\delta$, one can rather control the signal-to-noise ratio $\delta^{0} / \sigma$. CP proposed to let $\delta^{0}$ go to zero at a not too fast rate while letting $\sigma$ increase to infinity in a neighborhood of $T_{b}^{0}$. That is $\left(\delta_{T} / \sigma_{t}\right) \rightarrow 0$ at rate $O\left(T^{-1 / 2}\right)$ in a neighborhood of $T_{b}^{0}$. This is the same rate Elliott and Müller used for $\delta_{T} \rightarrow 0$. However, the difference here is that all the parameters in the models remain consistent. See CP for details. 


\section{Laplace-based Estimation}

We first formally define the Generalized Laplace (GL) estimator and introduce assumptions needed for the derivation of its large-sample properties. Section 4.2 describes the asymptotic framework adopted. The main results about the limit distribution of the estimate of $\lambda_{0}$ are presented in Section 4.3. Section 4.4 explains how to construct the GL estimator in practice.

\subsection{The Estimator and Additional Assumptions}

Using the properties of orthogonal LS projections, $\theta$ can be concentrated out from the objective function $Q_{h}\left(\theta, N_{b}\right)$, allowing us to dispense with the dependence of $Q_{h}\left(\theta, N_{b}\right)$ on $\theta$ and use $Q_{h}\left(N_{b}\right)$ hereafter. The parameter of interest is $N_{b}$, and the Quasi-posterior density $p_{h}\left(N_{b}\right)$ is given by,

$$
p_{h}\left(N_{b}\right) \triangleq \frac{\exp \left(Q_{h}\left(N_{b}\right)\right) \pi\left(N_{b}\right)}{\int_{\Gamma^{0}} \exp \left(Q_{h}\left(N_{b}\right)\right) \pi\left(N_{b}\right) d N_{b}},
$$

which constitutes a proper distribution over the parameter space $\Gamma^{0} \triangleq(0, N)$. The Quasiprior $\pi(\cdot)$ is a weight function or simply a prior probability density. For example, $\pi\left(N_{b}\right)=$ $d \mathbb{Q}\left(N_{b}\right) / d$ Leb $\left(N_{b}\right)$ for some probability distribution $\mathbb{Q}$, with Leb $(\cdot)$ the Lebesgue measure. The function $\pi(\cdot)$ satisfies weak regularity conditions. Following Chernozhukov and Hong (2003) we restrict attention to convex loss functions $l_{h}(\cdot)$. Common examples include, (i) $l_{h}(r)=a_{h}|r|^{m}$, the polynomial loss function with the squared loss function obtained when $m=2$, and the absolute deviation loss with $m=1$; (ii) $l_{h}(r)=a_{h}(\tau-\mathbf{1}(r \leq 0)) r$, the check loss function where $\left\{a_{h}\right\}$ is a positive sequence with $a_{h} \rightarrow \infty$. Given the Quasi-posterior density, we can define the expected risk under $p_{h}(\cdot)$, for the loss $l_{h}(\cdot)$, as $\mathcal{R}_{l, h}(s) \triangleq \mathbb{E}_{p_{h}}\left[l_{h}\left(s-\widetilde{N}_{b}\right)\right]$, where $\widetilde{N}_{b}$ is a random variable with distribution $p_{h}$ and $\mathbb{E}_{p_{h}}$ denotes expectation taken under $p_{h}$. Then,

$$
\mathcal{R}_{l, h}(s)=\int_{\Gamma^{0}} l_{h}\left(s-N_{b}\right)\left(\frac{\exp \left(Q_{h}\left(N_{b}\right)\right) \pi\left(N_{b}\right)}{\int_{\Gamma^{0}} \exp \left(Q_{h}\left(N_{b}\right)\right) \pi\left(N_{b}\right) d N_{b}}\right) d N_{b} .
$$

The GL estimator $\widehat{N}_{b}^{\text {GL }}$ is a decision rule that is least unfavorable given the information provided by the Quasi-posterior $p_{h}$ according to the loss function $l_{h}(\cdot)$. That is, $\widehat{N}_{b}^{\mathrm{GL}}$ minimizes the expected risk function in (4.2): $\widehat{N}_{b}^{\mathrm{GL}} \triangleq \operatorname{argmin}_{s \in \Gamma^{0}} \mathcal{R}_{l, h}(s)$. Observe that if $p_{h}\left(N_{b}\right)$ were a true posterior, then $\widehat{N}_{b}^{\mathrm{GL}}$ would naturally be viewed as a Bayesian estimator for the loss function $l_{h}(\cdot)$ and prior $\pi$. This suggests an interpretation of the Laplace-type estimator as a Quasi-Bayesian estimator.

In our setting, one can treat the density of the continuous record limit distribution of $\widehat{N}_{b}^{\mathrm{LS}}$ as the "Quasi-prior" $\pi$. As discussed below, the resulting Quasi-posterior in (4.1) provides useful information for inference about the parameter $N_{b}^{0}$ beyond that already included in the objective function $Q_{h}\left(N_{b}\right)$. Inference procedures based on the median of the Quasi-posterior density obtained 
when using the absolute loss function is a reasonable choice as we shall show. The GL estimation provides alternative inference methods that may be combined with the (frequentist) continuous record asymptotic framework, through the choice of $\pi$. Note that in order to construct such GL estimator with the continuous record Quasi-prior, one needs an estimate of the density of the continuous record distribution, computed as in CP; see Section 4.4 for details.

To understand why the GL estimation can be useful, consider Figure 1, which shows that when the magnitude of the break is small both the finite-sample and continuous record distribution display highly non-standard features. First, there are three modes; two are near the start and end of sample while the mode at the origin corresponds to the estimated break point. This multimodality signifies that there is a substantial tendency for the LS estimator to locate the break date towards the tails rather than close to the true break date. Second, the asymmetry in the density - which is always present unless the true break date is at mid-sample -implies that the span and actual location of the break matters for the precision of the estimator. CP documented that such features are still present to a lesser extent for moderate break sizes, although they disappear when they are large (cf. Figure 2). As opposed to simply relying on $Q_{h}\left(N_{b}\right)$ which is quite flat for small breaks, the GL estimation combines information from the continuous record density - through $\pi\left(N_{b}\right)$ - with information from the distribution of the criterion function to yield the Quasi-posterior. Given the highly non-standard features of the finite-sample distribution, the Quasi-posterior contains more accurate information about the uncertainty of the change-point. Hence, estimation and inference based on the latter may have better properties as we shall show.

Assumption 4.1. L denotes the set of functions $l: \mathbb{R} \rightarrow \mathbb{R}_{+}$that satisfy: $(i) l(r)$ is defined on $\mathbb{R}$, with $l(r) \geq 0$ and $l(r)=0$ if and only if $r=0$; (ii) $l(r)$ is continuous at $r=0$ but is not identically zero; (iii) $l(\cdot)$ is convex and $l(r) \leq 1+|r|^{m}$ for some $m>0$.

Assumption 4.2. The function $\pi: \mathbb{R} \rightarrow \mathbb{R}_{+}$is a continuous, uniformly positive density function satisfying $\pi^{0} \triangleq \pi\left(N_{b}^{0}\right)>0$, and $\pi^{0}<C_{\pi}$ for some finite $C_{\pi}$. Furthermore, $\pi\left(N_{b}\right)=0$ for all $N_{b} \notin \Gamma^{0}$, and $\pi$ is twice continuously differentiable in $N_{b}$ at $N_{b}^{0}$.

Definition 4.1. The random variable $\xi_{l}^{0}$ is uniquely defined by

$$
\Psi_{l}^{*}\left(\xi_{l}^{0}\right) \triangleq \inf _{s} \Psi_{l}^{*}(s)=\inf _{s} \int_{\Gamma^{*}} l(s-v)\left(e^{\mathscr{V}(v)} \pi\left(N_{b}^{0}+v / \vartheta\right) / \int_{\Gamma^{*}} e^{\mathscr{V}(w)} \pi\left(N_{b}^{0}+w / \vartheta\right) d w\right) d v
$$

where $\vartheta \triangleq\left\|\delta^{0}\right\| / \sigma^{2} ; \Gamma^{*}$ and the process $\mathscr{V}(\cdot)$ are specified below.

Lemma 4.1. For any $\eta>0$, there exists an $\epsilon>0$, such that

$$
\liminf _{h \downarrow 0} \mathbb{P}\left[\sup _{\left|N_{b}-N_{b}^{0}\right| \geq \eta} Q_{h}\left(N_{b}\right)-Q_{h}\left(N_{b}^{0}\right) \leq-\epsilon\right]=1 .
$$


The conditions in Assumption 4.1 are similar in spirit to those in Bickel and Yahav (1969) and Chernozhukov and Hong (2003). A convex loss function is usually employed in applications. The restriction imposed in part (iii) is not essential. What one needs is that the growth of the function $l(r)$ as $|r| \rightarrow \infty$ is slower than that of functions of the form $\exp (\epsilon|r|), \epsilon>0$. Further, note that part (iii) implicitly assumes that $l(\cdot)$ has been scaled by some constant for the inequality to hold. Assumption 4.2 on the Quasi-prior is rather mild. As anticipated above, one may take $\pi(\cdot)$ as the density of the limit process appearing in Proposition 3.2. The uniqueness of $\xi_{l}^{0}$ follows from the fact that there is a single break. Lemma 4.1 guarantees the identification of the parameters.

\subsection{Asymptotic Framework for the Generalized Laplace Estimation}

Our framework builds upon an expansion of the criterion function derived by CP under a continuous record. This is useful because it concentrates out the regression parameters and allows us to frame our theory in terms of a single local parameter $u=r_{h}\left(N_{b}-N_{b}^{0}\right)$, while the regression parameters are kept fixed at their true values; $r_{h}$ is some sample size-dependent sequence which converges to infinity and whose properties will be specified below. This makes $Q_{h}\left(\theta\left(N_{b}\right), N_{b}\right)=$ $\delta^{\prime}\left(N_{b}\right)\left(Z_{2}^{\prime} M_{X} Z_{2}\right) \delta\left(N_{b}\right)$ a function only of the localized break date $u=r_{h}\left(N_{b}-N_{b}^{0}\right)$. Because there is a single break in the model, it follows that the unknown parameter $N_{b}^{0} \in \Gamma^{0} \subset(0, N)$ is the unique maximizer of $Q_{0, h}\left(\theta\left(N_{b}\right), N_{b}\right)=\mathbb{E}\left[Q_{h}\left(\theta\left(N_{b}\right), N_{b}\right)-Q_{h}\left(\theta\left(N_{b}^{0}\right), N_{b}^{0}\right)\right]$. Further, let $\bar{Q}_{h}\left(\theta\left(N_{b}\right), N_{b}\right) \triangleq Q_{h}\left(\theta\left(N_{b}\right), N_{b}\right)-Q_{h}\left(\theta\left(N_{b}^{0}\right), N_{b}^{0}\right)$ and $G_{h}\left(\theta\left(N_{b}\right), N_{b}\right) \triangleq \bar{Q}_{h}\left(\theta\left(N_{b}\right), N_{b}\right)-$ $Q_{0, h}\left(\theta\left(N_{b}\right), N_{b}\right)$. These expansions of the criterion function shows that $G_{h}, \bar{Q}_{h}$ and $Q_{0, h}$ do not depend on $\theta\left(N_{b}\right)$ but only on $\theta^{0}$. Hence, since $\theta^{0} \in \mathbf{S} \subset \mathbb{R}^{p+q} \times \mathbb{R}^{q}$ is given, we shall omit it from the arguments of $G_{h}, \bar{Q}_{h}$ and $Q_{0, h}$ in what follows. ${ }^{3}$ The GL estimator $\widehat{N}_{b}^{\text {GL }}$ can equivalently be defined as the minimizer of

$$
\Psi_{l, h}(s)=\int_{\Gamma^{0}} l\left(s-N_{b}\right) \frac{\exp \left(\gamma_{h} \bar{Q}_{h}\left(N_{b}\right)\right) \pi\left(N_{b}\right)}{\int_{\Gamma^{0}} \exp \left(\gamma_{h} \bar{Q}_{h}\left(N_{b}\right)\right) \pi\left(N_{b}\right) d N_{b}} d N_{b}
$$

where $\left\{\gamma_{h}\right\}$ is a sequence which normalizes the sample criterion. Conditions on $\left\{\gamma_{h}\right\}$ will be stated below. The main theoretical result of this section concerns the large-sample properties of the GL estimator, which we derive as follows. We first show the convergence of the marginal distributions of the sample function $\Psi_{l, h}(s)$ to the marginal distributions of the random function $\Psi_{l}^{*}(s)=$ $\int_{\Gamma^{*}} l(s-v) \exp (\mathscr{V}(v)) \pi\left(N_{b}^{0}+v / \vartheta\right) /\left(\int_{\Gamma^{*}} \exp (\mathscr{V}(w)) \pi\left(N_{b}^{0}+w / \vartheta\right) d w\right) d u$, where $\pi_{h}(v)$ is defined below. Let $\mathbf{K} \triangleq\{s \in \mathbb{R}:|s| \leq K<\infty\}$. Next, we show that the family of probability measures in $\mathbb{C}_{b}(\mathbf{K})$ (the space of bounded continuous function from $\mathbf{K}$ into $\mathbb{R}$ ), generated by the contractions of $\Psi_{l, h}^{*}(s)$ on $\mathbf{K}$, is dense. As a final step, we analyze the oscillations of the minimum points of the sample criterion $\Psi_{l, h}^{*}(s)$. Given $u=r_{h}\left(N_{b}-N_{b}^{0}\right)$, we let $\pi_{h}(u) \triangleq \pi\left(N_{b}^{0}+u / r_{h}\right)$,

\footnotetext{
${ }^{3}$ The exact expressions for $G_{h}, \bar{Q}_{h}$ and $Q_{0, h}$ are provided at the beginning of Section A.2 of the supplement.
} 
$\widetilde{Q}_{h}(u) \triangleq \bar{Q}_{h}\left(N_{b}^{0}+u / r_{h}\right), \widetilde{G}_{h}(u) \triangleq G_{h}\left(N_{b}^{0}+u / r_{h}\right)$ and $\widetilde{Q}_{0, h}(u) \triangleq Q_{0, h}\left(N_{b}^{0}+u / r_{h}\right)$. Using the expression for $u$, we can apply a simple substitution in (4.4) which results in,

$$
\Psi_{l, h}(s)=\int_{\Gamma_{h}} l(s-u) \frac{\exp \left(\gamma_{h}\left(\widetilde{G}_{h}(u)+\widetilde{Q}_{0, h}(u)\right)\right) \pi_{h}(u) d u}{\int_{\Gamma_{h}} \exp \left(\gamma_{h}\left(\widetilde{G}_{h}(w)+\widetilde{Q}_{0, h}(w)\right)\right) \pi_{h}(w) d w}
$$

where $\Gamma_{h} \triangleq\left\{u \in \mathbb{R}: N_{b}^{0}+u / r_{h} \in \Gamma^{0}\right\}$. The local parameter $u$ introduced above depends on the normalizing factor $\left\{r_{h}\right\}$. We set $r_{h} \triangleq T^{1-\kappa} / \vartheta N, \kappa \in(0,1 / 2)$ with $\vartheta \triangleq\left\|\delta^{0}\right\|^{2} / \bar{\sigma}^{2}$ so as to have ${ }^{4}$

$$
u \triangleq \vartheta^{-1} T^{1-\kappa}\left(\lambda_{b}-\lambda_{0}\right)
$$

The factor $T^{1-\kappa}$ is the rate at which $N \widehat{\lambda}_{b}^{\mathrm{LS}}$ convergences to $N_{b}^{0}$ under the continuous record asymptotic setting. Note that the local parameter $u$ is allowed to vary over the entire real line. On the range $\{|u|<K\}, N_{b}$ approaches $N_{b}^{0}$ at the rate $T^{1-\kappa}$. Thus, we will deduce a convergence result for the normalized criterion function $\gamma_{h} \widetilde{Q}_{h}(u)$ toward a tight Gaussian process. For other values of $u$, we show that the tails of the Quasi-posterior are negligible. For the latter, one uses properties of the Gaussian component of the limit process $\mathscr{V}(\cdot)$, and show that it cannot diverge faster than the (negative) drift component. Since the Quasi-posterior is an exponential transform of the centered objective function, the tails of some expansion of the criterion function diverge (after rescaling) to minus infinity. This follows because the objective function can only be maximized for values of the parameter sufficiently close to $N_{b}^{0}$, and so its re-centered version is always negative and bounded away from zero if $N_{b}$ is far from $N_{b}^{0}$. The normalizing sequence $\left\{\gamma_{h}\right\}$ then makes the exponential transformation negligible for such $N_{b}$ far from $N_{b}^{0}$.

\subsection{Asymptotic Distribution of the Generalized Laplace Estimator}

The main result of this section is Theorem 4.1, which presents the limit distribution of the GL estimator for a general loss function $l(\cdot)$. As part of the proof, we show the weak convergence of $\widetilde{Q}_{h}(u)$ on the space of bounded functions from compact sets $\mathbf{B} \subset \mathbb{R}$ into $\mathbb{R}$, denoted by $\mathbb{D}_{b}(\mathbf{B})$. As a matter of notation let $\widetilde{\mathscr{W}}(\theta, u)$ denote an arbitrary sample process with bounded paths evaluated at $(\theta, u)$ with $u \in \mathbb{R}$. For each fixed $\theta$, we write $\widetilde{\mathscr{W}}(\theta, u) \Rightarrow \mathscr{W}(\theta, u)$ on the space $\mathbb{D}_{b}(\mathbf{B})$ if the process $\widetilde{\mathscr{W}}(\theta, \cdot)$ converges in law under the Skorohod metric to a process $\mathscr{W}(\theta, \cdot)$ defined on $\mathbb{D}_{b}(\mathbf{B})$. To simplify notation we omit the argument $\theta$ from the limit process. For general loss functions, the Laplace estimator is defined implicitly as the solution of a convex optimization problem. The theorem presents the limit distribution of the estimator under the change of time scale, discussed above. Formally, the limiting distribution is derived under a change of time scale $s \mapsto \psi_{h}^{-1} s$. Then, $t \triangleq \psi_{h}^{-1} s$ is the index on the new time scale. Hence, the sample criterion function

\footnotetext{
${ }^{4} N_{b}=N \lambda_{b}$ and so theoretical results about $\lambda_{b}$ translate immediately to $N_{b}$ (up to a constant $N$ ).
} 
$\widetilde{Q}_{h}(v)=\widetilde{G}_{h}(v)+\widetilde{Q}_{0, h}(v)$ will be shown to vary on $\Gamma^{*} \triangleq\left\{v \in \mathbb{R}:-\vartheta N_{b}^{0} \leq v \leq \vartheta\left(N-N_{b}^{0}\right)\right\}$ where $\vartheta=\left\|\delta^{0}\right\|^{2} \bar{\sigma}^{-2}$ and the optimization problem is then also defined with respect to $\Gamma^{*}$, namely via,

$$
\Psi_{l, h}^{*}(s) \triangleq \int_{\Gamma^{*}} l(s-u) \frac{\exp \left(\gamma_{h}\left(\widetilde{Q}_{h}(v)\right)\right) \pi_{h}(v) d v}{\int_{\Gamma^{*}} \exp \left(\gamma_{h}\left(\widetilde{Q}_{h}(w)\right)\right) \pi_{h}(w) d w}
$$

We next impose conditions on $\zeta_{k} \triangleq h^{-1} z_{k h} e_{k h}$ and $\gamma_{h}$ to derive the required limit distribution.

Assumption 4.3. Uniformly in $r \in[0,1],\left(T_{b}^{0}\right)^{-1 / 2} \sum_{k=1}^{\left\lfloor r T_{b}^{0}\right\rfloor} \zeta_{k} \Rightarrow \mathscr{W}_{1}(r),\left(T-T_{b}^{0}\right)^{-1 / 2} \sum_{k=T_{b}^{0}+1}^{T_{b}^{0}+\left\lfloor r\left(T-T_{b}^{0}\right)\right\rfloor}$ $\zeta_{k} \Rightarrow \mathscr{W}_{2}(r)$ where $\mathscr{W}_{i}(\cdot)$ is a multivariate Gaussian process on $[0,1]$ with zero mean and covariance $\mathbb{E}\left[\mathscr{W}_{i}(u), \mathscr{W}_{i}(s)\right]=\min \{u, s\} \Omega_{\mathscr{W}, i}(i=1,2), \Omega_{\mathscr{W}, 1} \triangleq \lim _{T \rightarrow \infty} \mathbb{E}\left[\left(T_{b}^{0}\right)^{-1 / 2} \sum_{k=1}^{T_{b}^{0}} \zeta_{k}\right]^{2}$ and $\Omega_{\mathscr{W}, 2} \triangleq$ $\lim _{T \rightarrow \infty} \mathbb{E}\left[\left(T-T_{b}^{0}\right)^{-1 / 2} \sum_{k=T_{b}^{0}+1}^{T} \zeta_{k}\right]^{2}$. For any $0<r_{0}<1$ with $r_{0} \neq \lambda_{0}, T^{-1} \sum_{k=\left\lfloor r_{0} T\right\rfloor+1}^{\left\lfloor\lambda_{0} T\right\rfloor} h^{-1} z_{k h} z_{k h}^{\prime} \stackrel{\mathbb{P}}{\rightarrow}$ $\left(\lambda_{0}-r_{0}\right) \Sigma_{Z, 1}$, and $T^{-1} \sum_{k=\left\lfloor\lambda_{0} T\right\rfloor+1}^{\left\lfloor r_{0} T\right\rfloor} h^{-1} z_{k h} z_{k h}^{\prime} \stackrel{\mathbb{P}}{\rightarrow}\left(r_{0}-\lambda_{0}\right) \Sigma_{Z, 2}$ with $\lambda_{-}$and $\lambda_{+}$, the minimum and maximum eigenvalues of the last two matrices with $0<\lambda_{-} \leq \lambda_{+}<\infty$.

Condition 1. As $T \rightarrow \infty, \gamma_{h} / T^{3 / 2-\kappa} \rightarrow \kappa_{\gamma}$ where $\kappa_{\gamma}>0$ is some constant.

Theorem 4.1. Let $l \in \boldsymbol{L}$ and Assumptions 2.1-2.6, 3.1-3.2, 4.1-4.3 as well as Condition 1 hold. Then, under the "fast time scale" as $T \rightarrow \infty$, we have $N\left(\widehat{\lambda}_{b}^{\mathrm{GL}}-\lambda_{0}\right) \Rightarrow \xi_{l}^{0}$, where $\xi_{l}^{0}$ is defined in Definition 4.1 with $\Gamma^{*}=\left(-\vartheta N_{b}^{0}, \vartheta\left(N-N_{b}^{0}\right)\right)$ and $\mathscr{V}$ from Proposition 3.2.

Corollary 4.1. Under the squared loss function $l_{h}(r)=a_{h}|r|^{2}$,

$$
N\left(\hat{\lambda}_{b}^{\mathrm{GL}}-\lambda_{0}\right) \Rightarrow \int_{\Gamma^{*}} v \frac{\exp (\mathscr{V}(v)) \pi\left(N_{b}^{0}+v / \vartheta\right)}{\int_{\Gamma^{*}} \exp (\mathscr{V}(w)) \pi\left(N_{b}^{0}+w / \vartheta\right) d w} d v
$$

in $\mathbb{D}_{b}(\mathbb{R})$ with $\mathscr{V}(v)$ defined in Proposition 3.2. Under a least-absolute loss function, $N\left(\widehat{\lambda}_{b}^{\mathrm{GL}}-\lambda_{0}\right)$ converges to the median of $\exp (\mathscr{V}(v)) \pi\left(N_{b}^{0}+v / \vartheta\right) / \int_{\Gamma^{*}} \exp (\mathscr{V}(w)) \pi\left(N_{b}^{0}+w / \vartheta\right) d w$.

Let $\rho \triangleq\left(\left(\delta^{0}\right)^{\prime}\langle Z, Z\rangle_{1} \delta^{0}\right)^{2} /\left(\left(\delta^{0}\right)^{\prime} \Omega_{\mathscr{W}, 1} \delta^{0}\right)$. After applying the usual change in variables [cf. Bai (1997), Bai and Perron (1998) and CP], we obtain a limit distribution expressed directly in terms of quantities that can be estimated.

Corollary 4.2. Let $l \in \boldsymbol{L}$ and Assumptions 2.1-2.6, 3.1-3.2, 4.1-4.3 and Condition 1 hold. Then, under the "fast time scale" and as $T \rightarrow \infty, N\left(\widehat{\lambda}_{b}^{\mathrm{GL}}-\lambda_{0}\right) \Rightarrow \xi_{l}^{0}$, with $\xi_{l}^{0}$ uniquely defined by

$$
\Psi_{l}^{*}\left(\xi_{l}^{0}\right)=\inf _{s} \Psi_{l}^{*}(s)=\inf _{s} \int_{\Gamma_{\rho}^{*}} l(s-v) \frac{\exp \left(\mathscr{V}^{*}(v)\right) \pi\left(N_{b}^{0}+v / \vartheta \rho\right)}{\int_{\Gamma_{\rho}^{*}} \exp \left(\mathscr{V}^{*}(w)\right) \pi\left(N_{b}^{0}+w / \vartheta \rho\right) d w} d v
$$

where $\Gamma_{\rho}^{*}=\left(-\vartheta \rho N_{b}^{0}, \vartheta \rho\left(N-N_{b}^{0}\right)\right)$ with $\mathscr{V}^{*}(\cdot)$ defined in Proposition 3.3. 
Theorem 4.1 states that the asymptotic distribution of the GL estimator-under the new "fast time scale" asymptotic framework - is an integral-ratio of functions of Gaussian processes. An advantage is that the knowledge of $\kappa$, which determines the rate of convergence on the original time scale, is not needed to conduct inference.

We can compare this limiting distribution with that of the Bayesian change-point estimator of Ibragimov and Has'minskiǐ (1981) [see their equation (2.17) on p. 338]. They considered maximum likelihood and Bayesian estimators of the change-point in a simple diffusion process. We note a few differences. First, the region of integration is $\Gamma^{*}$ instead of $\mathbb{R}$. Second, due to the change of time scale, the Quasi-prior $\pi(\cdot)$ enters the limiting distribution, a useful property in view of the features of the finite-sample distributions. It reflects the fact that the uncertainty in a change-point problem is often high enough that the information provided by the prior can influence the limiting behavior. Finally, note that the GL estimator conserves a classical (frequentist) interpretation.

\subsection{Construction of the GL Estimate}

The definition of the GL estimate involves a component, $\exp \left(Q_{h}\left(N_{b}\right)\right) / \int_{\Gamma^{0}} \exp \left(Q_{h}\left(N_{b}\right)\right) d N_{b}$, which is immediately available while the Quasi-prior $\pi\left(N_{b}\right)$ needs to be replaced by a consistent estimate. This requires to obtain by simulations an estimate of the density of the continuous record limiting distribution in (3.3). We follow CP. Theorem 3.2 shows that the limiting distribution of the LS break point estimator is related to the distribution of the location of the maximum of the process $\mathscr{V}(s)=\mathscr{W}(s)-\Lambda(s)$, or after a change in variable, of the process,

$$
\mathscr{V}^{*}(s)= \begin{cases}-\frac{|s|}{2}+W_{1}^{*}(-s), & \text { if } s<0 \\ -\frac{s}{2} \phi_{Z}+\phi_{e} W_{2}^{*}(s), & \text { if } s \geq 0\end{cases}
$$

where $W_{i}^{*}(i=1,2)$ are independent standard Wiener processes, $\phi_{Z} \triangleq\left(\delta^{0}\right)^{\prime} \Sigma_{Z, 2} \delta^{0} /\left(\delta^{0}\right)^{\prime} \Sigma_{Z, 1} \delta^{0}$ and $\phi_{e} \triangleq\left(\delta^{0}\right)^{\prime} \Omega_{\mathscr{W}, 2} \delta^{0} /\left(\delta^{0}\right)^{\prime} \Omega_{\mathscr{W}, 1} \delta^{0}$. Further, for every $t, s \in \mathbb{R}_{+}$, let $\Sigma^{0}(t, s) \triangleq \mathbb{E}(\mathscr{W}(t), \mathscr{W}(s))$. To conduct inference, one needs estimates for $N_{b}^{0}, \rho, \rho \vartheta N_{b}^{0}, \phi_{Z}$ and $\phi_{e}$. With the normalization $N=1, \hat{\lambda}_{b}^{\mathrm{LS}}=\widehat{T}_{b}^{\mathrm{LS}} / T$ is a natural estimate of $\lambda_{0}$. Consistent estimates of $\phi_{Z}$ and $\phi_{e}$ are given by

$$
\begin{aligned}
\widehat{\phi}_{Z} & =\frac{\widehat{\delta}^{\prime}\left(T-\widehat{T}_{b}^{\mathrm{LS}}\right)^{-1} \sum_{k=\widehat{T}_{b}^{\mathrm{LS}}+1}^{T} z_{k h} z_{k h}^{\prime} \widehat{\delta}}{\widehat{\delta}^{\prime}\left(\widehat{T}_{b}^{\mathrm{LS}}\right)^{-1} \sum_{k=1}^{\widehat{T}_{b}^{\mathrm{LS}}} z_{k h} z_{k h}^{\prime} \widehat{\delta}^{-1}} \\
\widehat{\phi}_{e} & =\frac{\widehat{\delta}^{\prime}\left(T-\widehat{T}_{b}^{\mathrm{LS}}\right)^{-1} \sum_{k=T_{b}^{\mathrm{LS}}+1}^{T} \widehat{e}_{k h}^{2} z_{k h} z_{k h}^{\prime} \widehat{\delta}}{\widehat{\delta}^{\prime}\left(\widehat{T}_{b}^{\mathrm{LS}}\right)^{-1} \sum_{k=1}^{\widehat{T}_{b}^{\mathrm{LS}}} \widehat{e}_{k h}^{2} z_{k h} z_{k h}^{\prime} \widehat{\delta}}
\end{aligned}
$$

where $\widehat{\delta}$ and $\widehat{e}_{k h}$ are the LS estimator of $\delta_{h}$ and the residuals. Let $\vartheta=\left\|\delta^{0}\right\|^{2} \bar{\sigma}^{-2} \rho$ and 


$$
\begin{aligned}
& \widehat{\vartheta}=\widehat{\rho}\|\widehat{\delta}\|^{2}\left(T^{-1} \sum_{k=1}^{T} \widehat{e}_{k h}^{2}\right)^{-1} \frac{\left(\widehat{\delta}^{\prime}\left(\widehat{T}_{b}^{\mathrm{LS}}\right)^{-1} \sum_{k=1}^{\widehat{T}_{b}^{\mathrm{LS}}} z_{k h} z_{k h}^{\prime} \widehat{\delta}\right)^{2}}{\widehat{\delta}^{\prime}\left(\widehat{T}_{b}^{\mathrm{LS}}\right)^{-1} \sum_{k=1}^{\widehat{T}_{b}^{\mathrm{LS}}} \widehat{e}_{k h}^{2} z_{k h} z_{k h}^{\prime} \widehat{\delta}} \\
& \widehat{\rho}=\frac{\left(\widehat{\delta}^{\prime}\left(\widehat{T}_{b}^{\mathrm{LS}}\right)^{-1} \sum_{k=1}^{\widehat{T}_{b}^{\mathrm{LS}}} z_{k h} z_{k h}^{\prime} \widehat{\delta}\right)^{2}}{\widehat{\delta}^{\prime}\left(\widehat{T}_{b}^{\mathrm{LS}}\right)^{-1} \sum_{k=1}^{\mathrm{T} \mathrm{LS}} \widehat{e}_{k h}^{2} z_{k h} z_{k h}^{\prime} \widehat{\delta}} .
\end{aligned}
$$

We have $\widehat{\vartheta} / h \stackrel{p}{\rightarrow} \vartheta$ and $\widehat{\rho} / h \stackrel{p}{\rightarrow} \rho$. The final step is to derive numerically the empirical counterpart of (4.8), i.e., to estimate the density of the continuous record limiting distribution (cf. Proposition 5.1 in $\mathrm{CP}$ ). The estimate is consistent under the conditions of Theorem 4.1 and under fixed shifts.

\section{Small-Sample Properties of the GL Estimators}

We conduct a Monte Carlo study to assess the small-sample accuracy of the GL estimator. We consider a least-absolute loss function with a continuous record prior (GL-CR) as well as a method based on the following iterative procedure which exploits Theorem 4.1. This variant, labeled GLCR-Iter, uses the median of the feasible density of the continuous record distribution evaluated at the GL-CR estimate instead of at $\widehat{N}_{b}^{\mathrm{LS}}$. Its justification is as follows. First, when the break size is small, the objective function is quite flat and highly variable. Hence, taking the median of the Quasi-prior instead of $p_{h}\left(N_{b}\right)$ is likely to involve less variability and more precise estimates. When the break size is large, $p_{h}\left(N_{b}\right)$ and the continuous record Quasi-prior have a similar shape with a peak at the same estimate. In principle, this iterative procedure may be based on any estimate $\widehat{N}_{b}$ such that $\widehat{N}_{b}=N_{b}^{0}+o_{p}(1)$. Furthermore, it can be viewed as an iterative version of the GL estimator as defined by (4.4) with $\gamma_{h} \rightarrow 0$. We also consider the least-squares (OLS) and the GL estimator under a least-absolute loss function with a uniform prior (GL-Uni). The least-squares estimator relies on a trimming parameter $\epsilon$. Common choices in applied work are $\epsilon=0.15,0.10$ and 0.05, which preclude locating the break in the first and last $100 \epsilon \%$ of the sample. Since the GL estimator uses the least-squares criterion function, it also involves the same trimming. Moreover, since it takes into account its whole distribution, the trimming parameter plays a relatively more important role. We recommend to set $\epsilon$ not too high, otherwise the GL estimator tends to be too much concentrated toward the middle of the sample for small breaks. Thus, we set $\epsilon=0.05$. We compare the mean absolute error (MAE), standard deviation (Std), root-mean-squared error (RMSE), and the $25 \%$ and $75 \%$ quantiles. 
We consider discrete-time DGPs which take the following form:

$$
y_{t}=D_{t} \varrho^{0}+Z_{t} \delta_{1}^{0}+Z_{t} \delta^{0} \mathbf{1}_{\left\{t>T_{b}^{0}\right\}}+e_{t}, \quad t=1, \ldots, T
$$

with $T=100$. Three versions of (5.1) are investigated: M1 is a partial structural change model with $\left\{Z_{t}\right\}$ a zero-mean stationary Gaussian $\mathrm{AR}(1)$ with autoregressive coefficient 0.3 and unit innovation variance, $D_{t}=1$ for all $t, \varrho^{0}=1$ and $\left\{e_{t}\right\}$ i.i.d. $\mathscr{N}(0,1.21)$ disturbances independent of $\left\{Z_{t}\right\}$; M2 involves a break in the mean which corresponds to $Z_{t}=1$ for all $t, D_{t}$ absent, and zero-mean stationary Gaussian $\operatorname{AR}(1)$ disturbances $\left\{e_{t}\right\}$ with $\mathrm{AR}$ coefficient 0.6 and innovation variance 0.49; M3 is a model with a lagged dependent variable with $D_{t}=y_{t-1}, Z_{t}=1, e_{t} \sim$ i.i.d. $\mathscr{N}(0,0.5)$, $\varrho^{0}=0.6$ and $Z_{t} \delta^{0} \mathbf{1}_{\left\{t>T_{b}^{0}\right\}}$ replaced by $Z_{t}\left(1.4 \varrho^{0} \delta^{0} \mathbf{1}_{\left\{t>T_{b}^{0}\right\}}\right)$. We set $\delta_{1}^{0}=1$ for all DGPs except in M3 where $\delta_{1}^{0}=0$. We consider $\lambda_{0}=0.3$ and 0.5 , and $\delta^{0}=0.3,0.4,0.6$ and 1 . By symmetry, the case $\lambda_{0}=0.7$ is omitted to avoid repetitions.

The results are presented in Tables 1-3. We document that the LS estimator displays a large absolute bias (large MAE) when the size of the break is small, which increases as the true break point moves away from mid-sample. The GL estimators successfully reduce the absolute bias; when the break point is about mid-sample the reduction in MAE is roughly $50 \%$ when the size of the break is small or moderate. It is interesting to note that the distributions of GL-CR and GL-CR-Iter are more concentrated around mid-sample than the distribution of the OLS estimator which locates nontrivial mass in the tails. This explains the large reduction in MAE when the break date is about mid-sample. When the break date is close to the tails the GL estimators still perform better than OLS, though the margin is smaller. This feature arises because for small breaks the objective function is quite flat with a small peak at the least-squares estimate while the resulting Quasi-posterior inherits some trimodality from the continuous record asymptotic distribution. Since higher mass is located close to the least-squares estimate - which corresponds to the middle mode - and less in the tails, the GL estimator tends to concentrate toward midsample. Therefore, it is useful to consider choosing a smaller trimming parameter so as not to miss the information contained in the tails of the distribution and to avoid the GL concentrating too much toward mid-sample. The smaller is $\epsilon$, the greater is the information used by the GL method to locate the break date. When the size of the break is large (i.e., $\delta^{0}=1$, bottom panel), although the absolute bias of the LS estimator becomes relatively small, the GL estimators still have lower MAE. Notably, the GL-type estimators display smaller variances than the LS estimator which leads to smaller RMSE. Thus, the GL estimate based on the CR prior dominates the LS estimator in both MAE and RMSE sense. Finally, the GL estimator that does not use the continuous record prior (i.e., GL-Uni), has a performance similar to the LS estimator since the Quasi-posterior contains essentially the same information about the objective function. Hence, using the continuous record prior is indeed important. This suggests that our asymptotic theory in Theorem 4.1 provides 
a useful approximation since it states that the prior adds useful information even in the limit. Comparing GL-CR and GL-CR-Iter, we note that their performance is similar, though the former often seems to be slightly more precise especially when the break is near mid-sample.

We now discuss how the GL estimator achieves more precision relative to LS. As shown in Figures 1-2 the finite-sample distribution of the LS estimator displays trimodality when the magnitude of the break is small. This occurs because when the evidence for a break is weak, the LS estimator has a tendency to locate the break in the tails as confirmed by the quantiles which show for example that the $75 \%$ quantile for the LS estimator is much larger than for the GL estimators. This is a quite undesirable property given that the break is assumed to be, e.g., in the middle $70 \%$ of the sample if $\epsilon=0.15$. The GL estimator does not share this property because it tends to concentrate more mass in the middle $70 \%$ of the sample. This works better when the true break date is about mid-sample. When it is near the tails, however, the choice $\epsilon=0.05$ avoids the distribution being too concentrated near mid-sample and be more evenly spread. What is important is that as $\delta^{0}$ increases we should expect the empirical distribution to move toward-and eventually concentrates about - the true break date. This is indeed achieved by the GL estimators.

\section{Inference Methods based on the GL Estimate}

In this section, we show how one can use the asymptotic properties of the GL estimator in order to extend the inference procedures for the break date presented in CP. We discuss several methods relying on the concept of highest density region. We begin with a description of this concept in Section 6.1. We present the new methods in Section 6.2. Section 6.3 provides the relevant theory.

\subsection{Highest Density Regions}

CP proposed to construct confidence sets using the concept of highest density region (HDR). The limit probability distribution of the break date estimator is given by (3.3), which is non-pivotal and depends on nuisance parameters. However, it can be simulated as described in Section 4.4. The next step is to derive numerically the regions of high density which can be used to construct the confidence sets for $T_{b}^{0}$. The method based on HDR is especially useful when the distribution of the estimate is multi-modal and/or asymmetric. When the density is symmetric and uni-modal, the HDR method reduces to the conventional one to construct confidence sets, i.e., symmetric and based on standard errors. CP discussed an algorithm to obtain the HDR following Hyndman (1996) [for more recent developments see Samworth and Wand (2010) and Mason and Polonik

(2008, 2009)]. Choose some $0<\alpha<1$ and let $\widehat{\mathbb{P}}_{T_{b}}^{\mathrm{CR}}$ denote the empirical counterpart of the limit distribution in Proposition 3.3 and $\widehat{\mu}^{\mathrm{CR}}$ its corresponding density function. 
Definition 6.1. Highest Density Region: Assume that the density function $f_{Y}(y)$ of a random variable $Y$ defined on a probability space $\left(\Omega_{Y}, \mathscr{F}_{Y}, \mathbb{P}_{Y}\right)$ and taking values on the measurable space $(\mathcal{Y}, \mathscr{Y})$ is continuous and bounded. The $(1-\alpha) 100 \%$ HDR is a subset $\mathbf{S}\left(\kappa_{\alpha}\right)$ of $\mathcal{Y}$ defined as $\mathbf{S}\left(\kappa_{\alpha}\right)=\left\{y: f_{Y}(y)>\kappa_{\alpha}\right\}$ where $\kappa_{\alpha}$ is the largest constant that satisfies $\mathbb{P}_{Y}\left(Y \in \mathbf{S}\left(\kappa_{\alpha}\right)\right) \geq 1-\alpha$.

Definition 6.2. OLS-CR HDR-based Confidence Sets for $T_{b}^{0}$ : A $(1-\alpha) 100 \%$ HDR-based confidence set for $T_{b}^{0}$ is a subset of $\{1, \ldots, T\}$ given by $C\left(\operatorname{cv}_{\alpha}\right)=\left\{T_{b} \in\{1, \ldots, T\}: T_{b} \in \mathbf{S}\left(\operatorname{cv}_{\alpha}\right)\right\}$, where $\mathbf{S}\left(\mathrm{cv}_{\alpha}\right)=\left\{T_{b}: \widehat{\mu}^{\mathrm{CR}}>\mathrm{cv}_{\alpha}\right\}$ with $\mathrm{cv}_{\alpha}$ satisfying $\sup _{\mathrm{cv}_{\alpha} \in \mathbb{R}_{+}} \widehat{\mathbb{P}}_{T_{b}}^{\mathrm{CR}}\left(T_{b} \in \mathbf{S}\left(\mathrm{cv}_{\alpha}\right)\right) \geq 1-\alpha$.

The confidence set $C\left(\mathrm{cv}_{\alpha}\right)$ has a frequentist interpretation even though the concept of HDR is often encountered in Bayesian analyses since it associates naturally to the derived posterior distribution, especially when the latter is multi-modal. Another feature of the confidence set $C\left(\mathrm{cv}_{\alpha}\right)$ is that it may consist of the union of several disjoint intervals when the size of the shift is small. In summary, one needs to carry out the following steps to construct the HDR-based confidence sets when using the LS estimate, labeled OLS-CR.

\section{Algorithm 1. OLS-CR HDR-based Confidence sets for $T_{b}^{0}$}

(1) Estimate by LS the date of the break and the regression coefficients from model (2.5); (2) Replace the break date $N_{b}^{0}$ by $\widehat{N}_{b}^{\mathrm{LS}}$, and the other quantities appearing in (3.3) by corresponding estimates as explained in Section 4.4; (3) Simulate the limit distribution $\widehat{\mathbb{P}}_{T_{b}}^{C R}$ from (3.3); (4) Compute the HDR of the empirical distribution $\widehat{\mathbb{P}}_{T_{b}}^{\mathrm{CR}}$ and include the point $T_{b}$ in the level $(1-\alpha)$ confidence set $C\left(\mathrm{cv}_{\alpha}\right)$ if $T_{b}$ satisfies the conditions in Definition 6.2.

\subsection{Confidence Sets based on $\widehat{N}_{b}^{\mathrm{GL}}$ with $\pi\left(N_{b}\right)=\widehat{\mu}^{\mathrm{CR}}\left(N_{b}\right)$}

We use the definition in (4.1) with the Quasi-prior $\pi\left(N_{b}\right)$ given by the continuous record asymptotic density $\widehat{\mu}^{\mathrm{CR}}$. We introduce the concept of Highest Quasi-posterior Density (HQPD) regions, defined analogously to HDR in Definition 6.1 with $p_{h}\left(N_{b}\right)$ being the object of interest. We then construct the confidence sets for $T_{b}^{0}$ as follows. We label this method by GL-CR.

\section{Algorithm 2. GL-CR HQPD-based Confidence sets for $T_{b}^{0}$}

(1) Estimate by least-squares $N_{b}^{0}$ and the regression coefficients from model (2.4); (2) Replace the break date $N_{b}^{0}$ by $\widehat{N}_{b}^{\mathrm{LS}}$, and the other quantities appearing in (3.3) by corresponding estimates as explained in Section 4.4; (3) Simulate the limit distribution $\widehat{\mathbb{P}}_{T_{b}}^{\mathrm{CR}}$ from (3.3) and set the Quasi-prior $\pi\left(N_{b}\right)$ equal to the probability density of $\widehat{\mathbb{P}}_{T_{b}}^{C R} ;(4)$ Construct the Quasi-posterior (4.1); (5) Obtain numerically the limit probability distribution of $\widehat{T}_{b}^{\mathrm{GL}}$ given in Corollary 4.2 and label it $\widehat{\mathbb{P}}_{T_{b}}^{\mathrm{GL}}$; (6) Compute the HQPD region of the probability distribution $\widehat{\mathbb{P}}_{T_{b}}^{\mathrm{GL}}$ and include the point $T_{b}$ in the level $(1-\alpha) \%$ confidence set $C_{\mathrm{GL}-\mathrm{CR}}\left(\mathrm{cv}_{\alpha}\right)$ if $T_{b}$ satisfies the conditions in Definition 6.2. 
The confidence set $C_{\mathrm{GL}-\mathrm{CR}}\left(\mathrm{cv}_{\alpha}\right)$ may also be interpreted as a Quasi-Bayes confidence set. Step 5 involves the derivation of the empirical counterpart of the limiting distribution of the GL estimator. In step 3, we use the continuous record Quasi-prior. Any prior $\pi\left(N_{b}\right)$ satisfying Assumption 4.2 could in principle be used, in which case the first three steps would not be needed. As discussed in Section 5, Theorem 4.1 also offers the possibility to construct alternative methods based on an iterative procedure as follows.

\section{Algorithm 3. GL-CR-Iter HDR Confidence sets for $T_{b}^{0}$}

(1) Carry out steps 1-3 in Algorithm 2; (2) Construct the Quasi-posterior in (4.1) and compute the estimate $\widehat{N}_{b}^{\mathrm{GL}}$; (3) Replace the break date $N_{b}^{0}$ by $\widehat{N}_{b}^{\mathrm{GL}}$, and the other quantities appearing in (3.3) by estimates as explained in Section 4.4; (4) Simulate the empirical counterpart of the probability distribution from Proposition 3.3 using step 3 labeled $\widehat{\mathbb{P}}_{T_{b}}^{\mathrm{CR}-\mathrm{GL}}$; (5) Compute the HDR of the empirical distribution $\widehat{\mathbb{P}}_{T_{b}}^{\mathrm{CR}-\mathrm{GL}}$ and include $T_{b}$ in the level $(1-\alpha)$ confidence set $C_{\mathrm{GL}-\mathrm{CR}-\mathrm{Iter}}\left(c_{\alpha}\right)$ if it satisfies the conditions in Definition 6.2.

\subsection{Theoretical Results on Inference Methods}

As explained above one generally needs estimates of some population quantities appearing in the continuous record asymptotic distribution in (3.2) and in the asymptotic distribution of the GL estimator. They can be constructed as explained in Section 4.4.

Assumption 6.1. Let $\widehat{\Sigma}_{i, h}(\cdot)$ be based on the estimates $\widehat{N}_{b, h}, \widehat{\delta}_{h}, \widehat{\phi}_{Z, h}$, and $\widehat{\phi}_{e, h}$ discussed in Section 4.4. For all $t, s \in \mathbb{R}$ and any $c>0, \widehat{\Sigma}_{i, h}(\cdot)$ satisfies, for $i=1,2:(i) \widehat{\Sigma}_{i, h}(t, s)=\Sigma_{i}^{0}(t, s)+o_{\mathbb{P}}(1)$; (ii) $\widehat{\Sigma}_{i, h}(t, s)=\widehat{\Sigma}_{i, h}(t, t)$ if $t<s$ and $\widehat{\Sigma}_{i, h}(t, s)=\widehat{\Sigma}_{i, h}(s, s)$ if $t>s$; (iii) $\widehat{\Sigma}_{i, h}(c t$, ct) $c \widehat{\Sigma}_{i, h}(t, t) ;(i v) \mathbb{E}\left\{\sup _{t=1} \widehat{\Sigma}_{i, h}^{2}(t, t)\right\}=O(1)$.

Assumption 6.1-(i) requires the availability of certain consistent estimators. Let $\left\{\widehat{\mathscr{W}}_{h}\right\}$ be a (sample-size dependent) sequence of two-sided Gaussian processes with covariance $\widehat{\Sigma}_{h}$. By Assumption 6.1-(i), the limit law of $\left\{\widehat{\mathscr{W}}_{h}\right\}$ is the same as the law of $\mathscr{W}$. Construct the process $\widehat{\mathscr{V}}_{h}$ by replacing the population quantities in $\mathscr{V}^{*}$ and replacing $\mathscr{W}$ by $\widehat{\mathscr{W}_{h}}$. Parts (ii)-(iv) are technical conditions needed for the integrability of $\exp \left(\widehat{\mathscr{V}}_{h}(\cdot)\right)$ and to prove the tightness of $\left\{\widehat{\mathscr{W}}_{h}\right\}$. Introduce the following random sample quantity:

$$
\widehat{\Psi}_{l, h}(s) \triangleq \int_{\widehat{\Gamma}^{*}} l(s-v) \frac{\exp \left(\widehat{\mathscr{V}}_{h}(v)\right) \pi\left(\widehat{N}_{b}^{\mathrm{GL}}+v\right)}{\int_{\widehat{\Gamma}^{*}} \exp \left(\widehat{\mathscr{V}}_{h}(w)\right) \pi\left(\widehat{N}_{b}^{\mathrm{GL}}+w\right) d w} d v
$$

where $\widehat{\Gamma}^{*}$ uses the estimates in Assumption 6.1 instead of the true values. Further, let $\widehat{\xi}_{l, h}$ be the absolute minimum point of $\widehat{\Psi}_{l, h}(s)$. Most inference methods introduced in the previous sub-section involve a numerical simulation of $\widehat{\Psi}_{l, h}$ or the moments or functions of the Quasi-posterior. The following theorem shows that $\widehat{\xi}_{l, h}$ converges in distribution to $\xi_{l}^{0}$. 
Theorem 6.1. Let $l \in \boldsymbol{L}$. Under Assumptions 2.1-2.6, 4.1-4.3 and 6.1, the distribution of $\widehat{\xi}_{l, h}$ is first-order equivalent to the distribution of $\xi_{l}^{0}$ in Theorem 4.1.

By Theorem 6.1, $\widehat{\xi}_{l, h}$ can be used to conduct statistical inference. For example, in order to use the GL HQDR-based method, step 5 of Algorithm 2 requires the derivation of the empirical counterpart of $\xi_{l}^{0}$. Theorem 6.1 shows the validity of methods based on numerically evaluating $\widehat{\xi}_{l, h}$. This is achieved by simulating $\widehat{\Psi}_{l, h}$ using of the estimators in Assumption 6.1. Since $\widehat{\xi}_{l, h} \Rightarrow \xi_{l}^{0}$, inference based on the probability distribution of $\widehat{\xi}_{l, h}$ is asymptotically valid.

\section{Small-Sample Evaluation of the GL Confidence Sets}

In this section, we compare the performance of the proposed confidence sets about $T_{b}^{0}$ with existing methods. We consider Bai's (1997) approach based on long-span shrinkage asymptotic arguments, Elliott and Müller's (2007) approach based on inverting Nyblom's (1989) statistic, the ILR method proposed by Eo and Morley (2015) based on the results in Qu and Perron (2007) and the recent HDR method proposed in CP based on the continuous record asymptotics with the least-squares estimate (OLS-CR). ${ }^{5}$ For brevity, we refer the readers to CP and Chang and Perron (2018) for a review and comprehensive evaluation of the first three methods. A brief summary is as follows. The empirical coverage probabilities of the confidence intervals obtained from Bai's (1997) method are often below the nominal level when the size of the break is small. This feature is not present for the other methods. Elliott and Müller's (2007) approach is by far the best one among existing methods in achieving an exact coverage rate that is the closest to the nominal level. However, this comes at the cost of lengths of the confidence sets which are always substantially larger relative to the other methods. This holds true across all break magnitudes. In addition, in models with serially correlated errors or lagged dependent variables, the length of the confidence set approaches the whole sample as the magnitude of the break increases. The ILR has coverage rates often above the nominal level and an average length significantly longer than the OLS-CR method at least when the magnitude of the shift is small or moderate. Further, the ILR does not work well when the errors are heteroskedastic and the regressor display serial correlation.

The recent OLS-CR method proposed in CP was shown to provide adequate empirical coverage probabilities over a wide range of data-generating mechanisms and across all break sizes and/or location of the break. The average length of the confidence sets is always shorter than that obtained with Elliott and Müller's (2007) method. The OLS-CR method delivers confidence sets with lengths only slightly larger than Bai's (1997) and the differences get smaller as the size of the break increases. Overall, the OLS-CR method strikes a better balance between accurate coverage

\footnotetext{
${ }^{5}$ Recently, Elliott et al. (2015) proposed a new test for structural breaks aimed at improving upon EM's approach. However, they did not propose methods for the inversion of such test to construct confidence intervals for the break date. Hence, we cannot evaluate their method.
} 
probabilities and length of the confidence sets. The numerical analysis in this section documents that the confidence sets derived from the GL inference are even more precise in terms of coverage probability than those from the OLS-CR method and the average length is always substantially shorter than that from Elliott and Müller's (2007).

We consider the same DGPs as in Section 5. To construct the OLS-CR method we follow the steps outlined in the previous section (see also CP for more details on the procedure for models with predictable processes). For model M2, to estimate the long-run variance we use Andrews' (1991) method along with Andrews and Monahan's (1992) AR(1) pre-whitened two-stage procedure to select the bandwidth. We consider the version $\widehat{U}_{T}\left(T_{\mathrm{m}}\right)$.neq of Elliott and Müller (2007) that allows for heteroskedastic regimes; using the restricted version when applicable leads to similar results.

The least-squares estimation method is employed with a trimming parameter $\epsilon=0.15$ and we use the required degrees of freedom adjustment for the statistic $\widehat{U}_{T}$ of Elliott and Müller (2007). The OLS-CR method and the methods proposed here do not involve any trimming and the confidence sets can potentially include any observation. The significance level is set at $\alpha=0.05$, and the break date occurs at $\left\lfloor T \lambda_{0}\right\rfloor$, where $\lambda_{0}=0.2,0.35,0.5$. The results for the $95 \%$ nominal coverage rates are presented in Tables 4-6. Each column reports the exact coverage rate and average length for a given break size $\delta^{0}$. The last row of each panel includes the rejection probability of a 5\%-level sup-Wald test using the asymptotic critical value of Andrews (1993); it serves as a statistical measure about the magnitude of the break.

Overall, the simulation results confirm previous findings about the performance of existing methods. Bai's (1997) method has a coverage rate below the nominal coverage level when the size of the break is small. For example, in model M2 for which there is high serial correlation in the disturbances, it fails to display a coverage rate above $90 \%$ even for moderate break sizes. In contrast, the method of Elliott and Müller (2007) overall yields very accurate empirical coverage rates. However, the average length of the confidence intervals is systematically much larger than those from all other methods across all DGPs, break sizes and break locations. ${ }^{6}$

The OLS-CR method provides good coverage rates for all break magnitudes and break locations. This holds even when there is high serial correlation in the errors (cf. M2). Furthermore, the confidence sets have average lengths significantly shorter than those from Elliott and Müller (2007). Thus, the OLS-CR method strikes overall a good balance between accurate coverage rates and length of the confidence sets. As observed in CP, this method tends to display an empirical coverage rate slightly below $95 \%$ for particular DGPs when the break size is small.

For the GL-based methods, we consider GL-CR and GL-CR-Iter implemented with the least-

\footnotetext{
${ }^{6}$ This problem is more severe when the errors are serially correlated or the model includes lagged dependent variables. Regarding the former, this in part may be due to issues with Newey and West HAC-type estimators when there are structural breaks [see Casini (2018, 2019a, 2019b), Casini and Perron (2019), Chang and Perron (2018), Crainiceanu and Vogelsang (2007), Deng and Perron (2006), Fossati (2018), Juhl and Xiao (2009), Kim and Perron (2009), Martins and Perron (2016), Perron and Yamamoto (2019) and Vogeslang (1999)].
} 
absolute deviation loss function. We do not report results with a uniform prior because as documented Section 5, it has poor finite-sample properties with respect to MAE and RMSE for small breaks. In general, the family of methods based on the GL estimator display better features compared to the OLS-CR method. GL-CR provides shorter length than GL-CR-Iter and OLS-CR, and it displays decent coverage rates. GL-CR-Iter provides coverage rates and lengths similar to OLS-CR, yet the coverage rates are more accurate than GL-CR. Thus, we find that the GL-CR-Iter is better than GL-CR if the primary goal is accurate coverage of the confidence sets.

In summary, the simulation results suggest that the GL-CR-Iter method using the GL estimates is reliable as it provides accurate coverage rates close to the nominal level and average lengths of the confidence sets shorter relative to existing methods developed under large- $N$ asymptotics.

\section{Concluding Remarks}

Building upon the continuous record asymptotic framework of Casini and Perron (2020a), we propose a Generalized Laplace (GL) procedure for the construction of the estimates and confidence sets for the date of a structural change. It is defined as the minimizer of the expected risk with the expectation taken under the Quasi-posterior, where the latter is constructed by applying a simple transformation to the least-squares criterion function. Our motivation stems from the nonstandard properties of the finite-sample distribution of the least-squares break point estimator. The advantage of the GL procedure is that it combines information from the LS estimate of the break point, the objective function and the continuous record distribution theory. The GL estimate is more precise than the usual LS estimate, lower MAE and RMSE, especially when the true break date is about middle sample. In order to achieve this result, it is advisable to choose a small trimming parameter (e.g., $\epsilon=0.05$ ). We also present inference methods that use the concept of HDR; the resulting confidence sets for the break date strike a better balance between empirical coverage rates and average lengths of the confidence sets relative to traditional long-span methods. Overall, among the GL procedures, we find that GL-CR provides more precise estimates for the break date while GL-CR-Iter results in more accurate coverage rates. 


\section{References}

Aït-Sahalia, Y., Jacod, J., 2014. High-frequency financial econometrics. Princeton University Press. Andersen, T.G., Fusari, N., Todorov, V., 2016. Short-term market risk implied by weekly options. Journal of Finance 72 (3), 1335-1386.

Andrews, D.W.K., 1991. Heteroskedasticity and autocorrelation consistent covariance matrix estimation. Econometrica 59, 817-858.

Andrews, D.W.K., 1993. Tests for parameter instability and structural change with unknown change-point. Econometrica 61 (4), 821-56.

Andrews, D.W.K., Monahan, J.C., 1992. An improved heteroskedasticity and autocorrelation consistent covariance matrix estimator. Econometrica 60 (4), 953-966.

Baek, Y., 2019. Estimation of structural break point in linear regression models. arXiv preprint arXiv 1811.03720.

Bai, J., 1997. Estimation of a change-point in multiple regression models. The Review of Economics and Statistics 79 (4), 551-563.

Bai, J., Perron, P., 1998. Estimating and testing linear models with multiple structural changes. Econometrica 66 (1), 47-78.

Bai, J., Perron, P., 2003. Computation and analysis of multiple structural changes. Journal of Applied Econometrics 18, 1-22.

Bandi, F. M., Renò, R., 2016. Price and volatility co-jumps. Journal of Financial Economics 119 (1), 107-146.

Barndorff-Nielsen, O.E., Shephard, N., 2004. Econometric analysis of realised covariation: high frequency based covariance, regression and correlation in financial economics. Econometrica $72(3), 885-925$.

Bickel, P.J., Yahav, J.A., 1969. Some contributions to the asymptotic theory of Bayes solutions. Zeitschrift für Wahrscheinlichkeitstheorie und Verwandte Gebiete 11 (4), 257-276.

Casini, A., 2018. Tests for forecast instability and forecast failure under a continuous record asymptotic framework. arXiv preprint arXiv:1803.10883.

Casini, A., 2019a. Improved methods for statistical inference in the context of various types of parameter variation. Ph.D dissertation, Boston University.

Casini, A., 2019b. Theory of evolutionary spectra for heteroskedasticity and autocorrelation robust inference in possibly misspecified and nonstationary models. Unpublished manuscript, Department of Economics and Finance, University of Rome Tor Vergata.

Casini, A., Perron, P., 2019. Structural breaks in time series. In: Oxford Research Encyclopedia of Economics and Finance. Oxford University Press.

Casini, A., Perron, P., 2020a. Continuous record asymptotics for structural change models. arXiv preprint arXiv:1803.10881.

Casini, A., Perron, P., 2020b. Generalized Laplace inference in multiple change-points models. arXiv preprint arXiv:1803.10871.

Casini, A., Perron, P., 2020c. Supplement to continuous record Laplace-based inference in structural change models. Unpublished manuscript, Department of Economics and Finance, University of Rome Tor Vergata.

Chambers, M.J., Taylor, A.M.R., 2019. Deterministic parameter change models in continuous and discrete time. Journal of Time Series Analysis.

Chang, S.Y., Perron, P., 2018. A comparison of alternative methods to construct confidence intervals for the estimate of a break date in linear regression models. Econometric Reviews 37 (6), 
577-601.

Chernozhukov, V., Hong, H., 2003. An MCMC approach to classical estimation. Journal of Econometrics $115(2), 293-346$.

Crainiceanu, C.M., Vogelsang, T.J., 2007. Nonmonotonic power for tests of a mean shift in a time series. Journal of Statistical Computation and Simulation 77 (6), 457-476.

Dempster, A.P., Laird, N.M., Rubin, D.B., 1977. Maximum likelihood from incomplete data via the EM algorithm. Journal of the Royal Statistical Society. Series B 39 (1), 1-38.

Deng, A., Perron, P., 2006. A comparison of alternative asymptotic frameworks to analyse a structural change in a linear time trend. Econometrics Journal 9 (3), 423-447.

Elliott, G., Müller, U.K., 2007. Confidence sets for the date of a single break in linear time series regressions. Journal of Econometrics 141 (2), 1196-1218.

Elliott, G., Müller, U.K., Watson, M.W., 2015. Nearly optimal tests when a nuisance parameter is present under the null hypothesis. Econometrica 83 (2), 771-811.

Eo, Y., Morley, J., 2015. Likelihood-ratio-based confidence sets for the timing of structural breaks. Quantitative Economics 6 (2), 463-497.

Forneron, J.J., Ng, S., 2018. The ABC of simulation estimation with auxiliary statistics. Journal of Econometrics 205 (1), 112-139.

Fossati, S., 2018. Testing for state-dependent predictive ability. Unpublished manuscript, Department of Economics, University of Alberta.

Hawkins, D., 1976. Point estimation of the parameters of piecewise regression models. Journal of Applied Statistics 25 (1), 51-57.

Hounyo, U., Liu, Z., Varneskov, R.T., 2020. Bootstrapping Laplace Transforms of Volatility. Unpublished manuscript.

Hyndman, R.J., 1996. Computing and graphing highest density regions. The American Statistician $50(2), 120-126$.

Ibragimov, A., Has'minskiř, R.Z., 1981. Statistical estimation: asymptotic theory. Springer-Verlag New York.

Jiang, L., Wang, X., Yu, S., 2017. In-fill asymptotic theory for structural break point in autoregression: A unified theory. Unpublish manuscript, School of Economics, Singapore Management University.

Jiang, L., Wang, X., Yu, S., 2018. New distribution theory for the estimation of structural break point in mean. Journal of Econometrics 205 (1), 156-176.

Juhl, T., Xiao, Z., 2009. Testing for changing mean with monotonic power. Journal of Econometrics 148 (1), 14-24.

Kim, D., Perron, P., 2009. Assessing the relative power of structural break tests using a framework based on the approximate Bahadur slope. Journal of Econometrics 149 (1), 26-51.

Laplace, P.S., 1774. Memoir on the probability of causes of events. Mémoires de Mathématique et de Physique Tome Sixième, (English translation by S. M. Stigler 1986. Statist. Sci., 1(19):364378).

Laredo, C., 1990. A sufficient condition for asymptotic sufficiency of incomplete observations of a diffusion process. Annals of Statistics 18 (3), 1158-1171.

Li, J., Todorov, V., Tauchen, G., 2017. Adaptive estimation of continuous-time regression models using high-frequency data. Journal of Econometrics 200 (1), 36-47.

Li, J., Xiu, D., 2016. Generalized method of integrated moments for high-frequency data. Econometrica 84 (4), 1613-1633.

Martins, L., Perron, P., 2016. Improved tests for forecast comparisons in the presence of instabili- 
ties. Journal of Time Series Analysis 37 (5), 650-659.

Mason, D.M., Polonik, W., 2008. Asymptotic normality of plug-in level set estimates, extended version.

Mason, D.M., Polonik, W., 2009. Asymptotic normality of plug-in level set stimates. Annals of Applied Probability 19 (3), 1108-1142.

Newey, W.K., West, K.D., 1987. A simple positive semidefinite, heteroskedastic and autocorrelation consistent covariance matrix. Econometrica 55, 703-708.

Nyblom, J., 1989. Testing for the constancy of parameters over time. Journal of the American Statistical Association 89 (451), 223-230.

Perron, P., 1991. A continuous-time approximation to the unstable first-order autoregressive process: the case without an intercept. Econometrica 59 (1), 211-236.

Perron, P., Qu, Z., 2006. Estimating restricted structural change model. Journal of Econometrics 134 (2), 373-399.

Perron, P., Yamamoto, Y., 2019. Testing for changes in forecast performance. Journal of Business and Economic Statistics forthcoming.

Phillips, P.C.B., 1987. Time series regression with a unit root. Econometrica 55 (2), 277-301.

Qu, Z., Perron, P., 2007. Estimating and Testing Structural Changes in Multivariate Regressions. Econometrica 75 (2), 459-502.

Samworth, R.J., Wand, M.P., 06 2010. Asymptotics and optimal bandwidth selection for highest density region estimation. Annals of Statistics 38 (3), 1767-1792.

Sorensen, M., Uchida, M., 12 2003. Small-diffusion asymptotics for discretely sampled stochastic differential equations. Bernoulli 9 (6), 1051-1069.

Vogeslang, T., 1999. Sources of nonmonotonic power when testing for a shift in mean of a dynamic time series. Journal of Econometrics 88 (2), 283-299. 


\section{A Appendix}
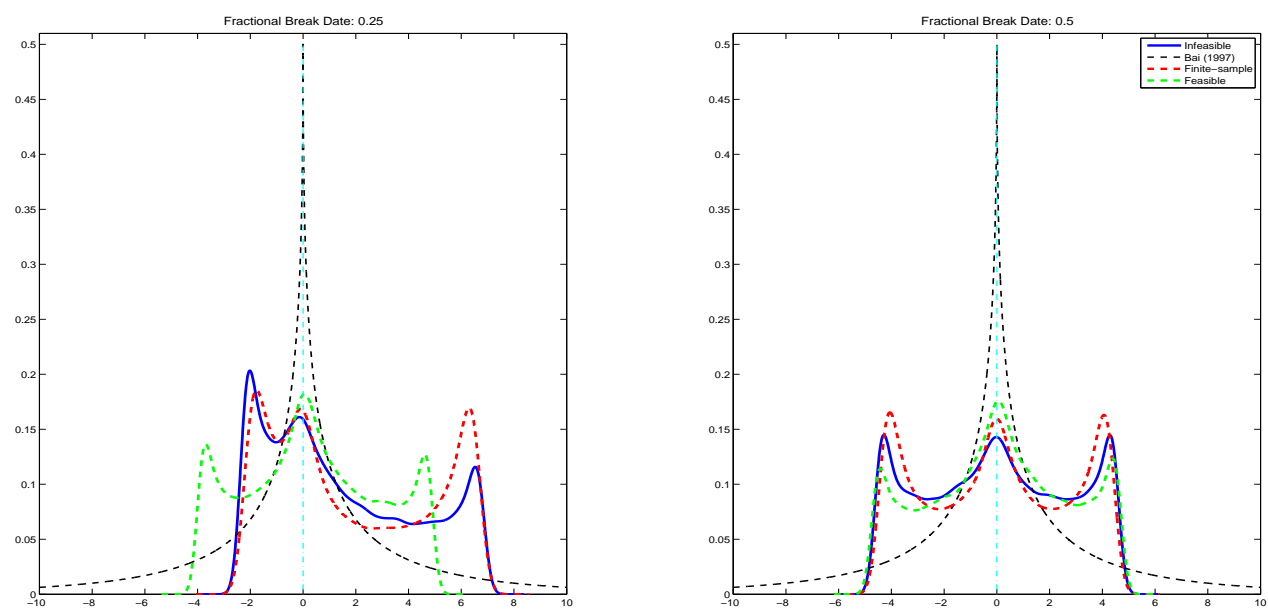

Figure 1: The probability density of the least-squares estimator $\widehat{T}_{b}-T_{b}^{0}$ for the model $y_{t}=\mu^{0}+Z_{t} \delta_{1}^{0}+$ $Z_{t} \delta^{0} \mathbf{1}_{\left\{t>\left\lfloor T \lambda_{0}\right\rfloor\right\}}+e_{t}, Z_{t}=0.3 Z_{t-1}+u_{t}-0.1 u_{t-1}, u_{t} \sim$ i.i.d. $\mathscr{N}(0,1), e_{t} \sim$ i.i.d. $\mathscr{N}(0,1),\left\{u_{t}\right\}$ independent from $\left\{e_{t}\right\}, T=100$ with break magnitude $\delta^{0}=0.3$ and true break point $\lambda_{0}=0.25$ and 0.5 (the left and right panel, respectively). The blue solid (resp., green broken) line is the density of the infeasible (reps., feasible) continuous record asymptotic distribution of $\mathrm{CP}$, the black broken line is the density of the asymptotic distribution from Bai (1997) and the red broken line break is the density of the finite-sample distribution.
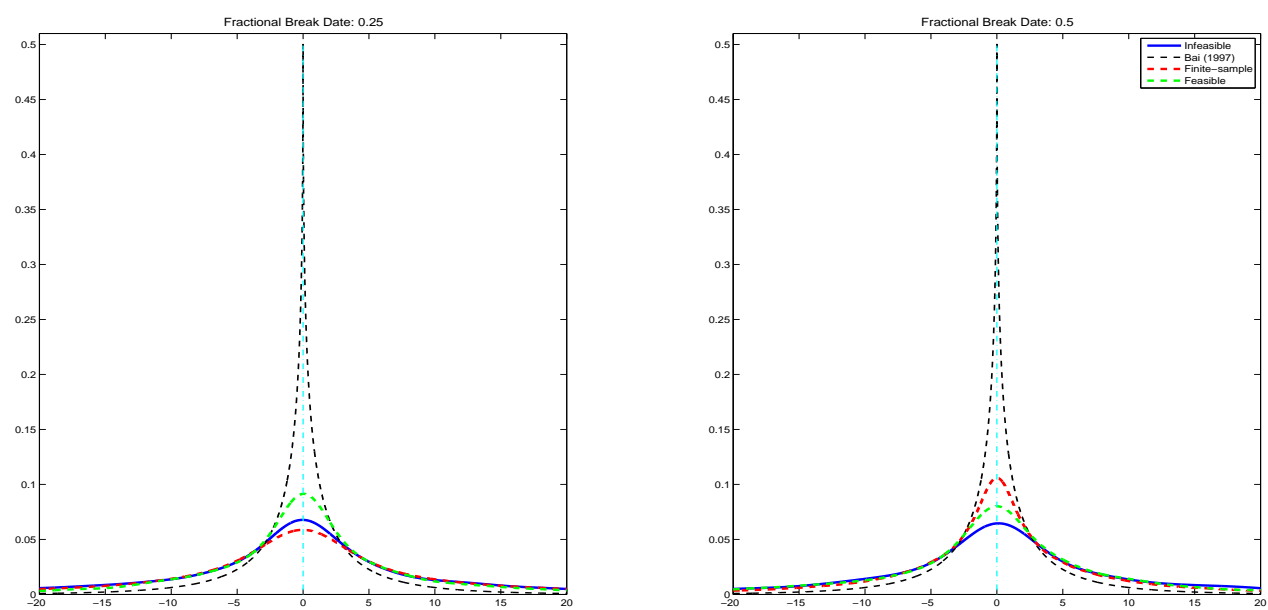

Figure 2: The comments in Figure 1 apply but with a break magnitude $\delta^{0}=1.5$. 
Table 1: Small-sample accuracy of the estimates of the break point $T_{b}^{0}$ for model M1

\begin{tabular}{|c|c|c|c|c|c|c|c|c|c|c|c|}
\hline \multirow{4}{*}{$\delta^{0}=0.3$} & \multirow[b]{3}{*}{ OLS } & MAE & Std & RMSE & $Q_{0.25}$ & $Q_{0.75}$ & MAE & Std & RMSE & $Q_{0.25}$ & $Q_{0.75}$ \\
\hline & & \multicolumn{5}{|c|}{$\lambda_{0}=0.3$} & \multicolumn{5}{|c|}{$\lambda_{0}=0.5$} \\
\hline & & 25.04 & 30.23 & 32.50 & 18 & 72 & 25.58 & 29.15 & 29.38 & 29 & 82 \\
\hline & GL-CR & 14.20 & 10.60 & 19.06 & 29 & 57 & 4.94 & 9.82 & 9.97 & 46 & 54 \\
\hline \multirow{6}{*}{$\delta^{0}=0.4$} & GL-CR-Iter & 17.32 & 22.64 & 22.64 & 29 & 59 & 8.16 & 9.66 & 9.69 & 41 & 59 \\
\hline & GL-Uni & 19.17 & 20.68 & 25.32 & 28 & 62 & 16.30 & 19.67 & 19.66 & 35 & 65 \\
\hline & OLS & 21.65 & 25.82 & 28.48 & 25 & 62 & 19.85 & 24.84 & 24.85 & 35 & 70 \\
\hline & GL-CR & 13.31 & 11.63 & 17.83 & 28 & 50 & 4.77 & 9.75 & 9.86 & 46 & 52 \\
\hline & GL-CR-Iter & 14.93 & 10.02 & 17.97 & 30 & 56 & 7.41 & 9.13 & 9.14 & 43 & 57 \\
\hline & GL-Uni & 16.22 & 19.94 & 22.89 & 27 & 55 & 14.46 & 18.18 & 18.17 & 37 & 63 \\
\hline \multirow[t]{4}{*}{$\delta^{0}=0.6$} & OLS & 12.95 & 19.92 & 20.68 & 26 & 38 & 11.69 & 17.10 & 17.10 & 44 & 56 \\
\hline & GL-CR & 12.25 & 12.51 & 15.56 & 29 & 49 & 4.46 & 8.82 & 8.64 & 49 & 51 \\
\hline & GL-CR-Iter & 11.99 & 8.56 & 14.72 & 30 & 44 & 5.23 & 7.13 & 7.14 & 46 & 54 \\
\hline & GL-Uni & 11.24 & 16.19 & 17.29 & 26 & 40 & 9.60 & 13.75 & 13.36 & 44 & 56 \\
\hline \multirow[t]{4}{*}{$\delta^{0}=1$} & OLS & 9.72 & 14.91 & 14.90 & 27 & 40 & 4.89 & 8.25 & 8.24 & 48 & 52 \\
\hline & GL-CR & 5.06 & 8.17 & 8.21 & 27 & 32 & 2.81 & 7.17 & 7.21 & 49 & 51 \\
\hline & GL-CR-Iter & 8.41 & 4.80 & 9.67 & 29 & 39 & 3.81 & 6.31 & 6.34 & 48 & 52 \\
\hline & GL-Uni & 4.69 & 8.35 & 8.36 & 27 & 32 & 4.51 & 7.47 & 7.47 & 48 & 52 \\
\hline
\end{tabular}

The model is $y_{t}=\varrho^{0}+Z_{t} \delta_{1}^{0}+Z_{t} \delta^{0} \mathbf{1}_{\left\{t>\left\lfloor T \lambda_{0}\right\rfloor\right\}}+e_{t}, Z_{t}=0.3 Z_{t-1}+u_{t}, u_{t} \sim$ i.i.d. $\mathscr{N}(0,1) e_{t} \sim$ i.i.d. $\mathscr{N}(0,1.21), T=100$. The columns refer to Mean Absolute Error (MAE), standard deviation (Std), Root Mean Squared Error (RMSE) and the $25 \%$ and $75 \%$ empirical quantiles. OLS is the least-squares estimator; GL-CR is the GL estimator under a least-absolute loss function with the continuous record prior; GL-CR-Iter is the median of the density of the continuous record distribution which uses the GL estimator in place of $\widehat{N}_{b}^{L S}$; GL-Uni is the GL estimator under a least-absolute loss function with a uniform prior. 
Table 2: Small-sample accuracy of the estimates of the break point $T_{b}^{0}$ for model M2

\begin{tabular}{|c|c|c|c|c|c|c|c|c|c|c|c|}
\hline \multirow{4}{*}{$\delta^{0}=0.3$} & \multirow[b]{3}{*}{ OLS } & MAE & Std & RMSE & $Q_{0.25}$ & $Q_{0.75}$ & MAE & Std & RMSE & $Q_{0.25}$ & $Q_{0.75}$ \\
\hline & & \multicolumn{5}{|c|}{$\lambda_{0}=0.3$} & \multicolumn{5}{|c|}{$\lambda_{0}=0.5$} \\
\hline & & 26.84 & 28.12 & 33.00 & 21 & 76 & 23.02 & 26.86 & 26.76 & 25 & 75 \\
\hline & GL-CR & 12.79 & 13.13 & 18.46 & 29 & 57 & 11.84 & 13.17 & 13.12 & 35 & 65 \\
\hline \multirow{5}{*}{$\delta^{0}=0.4$} & GL-CR-Iter & 14.47 & 10.29 & 20.21 & 28 & 58 & 8.76 & 10.01 & 10.24 & 41 & 59 \\
\hline & GL-Uni & 21.78 & 21.73 & 27.71 & 28 & 66 & 17.84 & 20.90 & 20.98 & 32 & 68 \\
\hline & OLS & 23.62 & 26.99 & 30.23 & 21 & 70 & 21.23 & 25.43 & 25.44 & 25 & 75 \\
\hline & GL-CR & 16.36 & 13.86 & 21.49 & 29 & 61 & 11.56 & 11.97 & 12.25 & 36 & 64 \\
\hline & GL-CR-Iter & 17.19 & 10.81 & 20.35 & 28 & 57 & 8.30 & 9.95 & 10.01 & 43 & 57 \\
\hline \multirow{3}{*}{$\delta^{0}=0.6$} & GL-Uni & 20.18 & 21.25 & 26.30 & 28 & 64 & 16.53 & 19.97 & 19.98 & 34 & 64 \\
\hline & OLS & 19.80 & 24.62 & 26.25 & 21 & 57 & 17.34 & 22.39 & 22.34 & 37 & 65 \\
\hline & GL-CR & 12.84 & 13.66 & 18.23 & 30 & 56 & 9.96 & 11.93 & 11.99 & 38 & 58 \\
\hline \multirow{6}{*}{$\delta^{0}=1$} & GL-CR-Iter & 14.85 & 11.52 & 17.56 & 29 & 52 & 7.26 & 9.20 & 9.22 & 44 & 55 \\
\hline & GL-Uni & 16.04 & 20.05 & 22.77 & 26 & 56 & 13.85 & 17.81 & 17.94 & 38 & 60 \\
\hline & OLS & 11.69 & 18.43 & 19.26 & 27 & 40 & 9.38 & 14.40 & 14.40 & 46 & 54 \\
\hline & GL-CR & 6.82 & 10.85 & 12.81 & 27 & 38 & 6.96 & 9.43 & 9.52 & 44 & 53 \\
\hline & GL-CR-Iter & 10.67 & 7.54 & 13.02 & 30 & 39 & 4.44 & 6.71 & 6.85 & 47 & 53 \\
\hline & GL-Uni & 9.44 & 14.60 & 15.15 & 27 & 37 & 8.17 & 12.34 & 12.34 & 45 & 54 \\
\hline
\end{tabular}

The model is $y_{t}=\delta_{1}^{0}+\delta^{0} \mathbf{1}_{\left\{t>\left\lfloor T \lambda_{0}\right\rfloor\right\}}+e_{t}, e_{t}=0.6 e_{t-1}+u_{t}, u_{t} \sim i . i . d . \mathscr{N}(0,0.49), T=100$. The notes of Table 1 apply.

Table 3: Small-sample accuracy of the estimates of the break point $T_{b}^{0}$ for model M3

\begin{tabular}{ccccccc|ccccc}
\hline \multirow{5}{*}{$\delta^{0}=0.3$} & & MAE & \multicolumn{1}{c}{ Std } & RMSE & $Q_{0.25}$ & $Q_{0.75}$ & MAE & \multicolumn{1}{c}{ Std } & \multicolumn{1}{c}{ RMSE } & $Q_{0.25}$ & $Q_{0.75}$ \\
\cline { 3 - 11 } & OLS & 22.33 & 26.93 & 29.88 & 25 & 43 & 17.23 & 22.54 & 22.48 & 36 & 64 \\
& GL-CR & 12.79 & 13.32 & 18.41 & 29 & 57 & 10.18 & 12.11 & 12.16 & 38 & 62 \\
& GL-CR-Iter & 13.92 & 15.42 & 19.05 & 29 & 56 & 10.85 & 13.62 & 13.69 & 39 & 61 \\
$\delta^{0}=0.4$ & GL-Uni & 15.92 & 16.81 & 21.40 & 30 & 55 & 11.28 & 14.54 & 14.54 & 41 & 59 \\
& OLS & 22.30 & 26.62 & 29.55 & 22 & 61 & 13.98 & 19.70 & 19.68 & 42 & 58 \\
& GL-CR & 10.08 & 12.73 & 16.23 & 29 & 46 & 9.08 & 11.41 & 11.41 & 42 & 58 \\
& GL-CR-Iter & 11.08 & 14.13 & 16.12 & 29 & 46 & 9.10 & 12.25 & 12.28 & 43 & 57 \\
$\delta^{0}=0.6$ & GL-Uni & 12.87 & 15.64 & 18.53 & 29 & 49 & 9.62 & 12.98 & 12.97 & 43 & 57 \\
& OLS & 8.56 & 15.91 & 16.15 & 27 & 33 & 8.04 & 13.24 & 13.22 & 46 & 54 \\
& GL-CR & 4.68 & 8.99 & 10.13 & 29 & 35 & 5.62 & 8.15 & 8.15 & 47 & 53 \\
& GL-CR-Iter & 7.06 & 10.90 & 11.35 & 29 & 35 & 6.17 & 9.36 & 9.36 & 46 & 53 \\
$\delta^{0}=1$ & GL-Uni & 7.77 & 11.85 & 12.79 & 28 & 38 & 6.40 & 9.63 & 9.62 & 46 & 54 \\
& OLS & 2.55 & 5.39 & 5.45 & 29 & 31 & 2.42 & 4.52 & 4.52 & 49 & 51 \\
& GL-CR & 1.24 & 3.26 & 3.48 & 29 & 31 & 2.31 & 4.11 & 4.11 & 49 & 51 \\
& GL-CR-Iter & 2.06 & 5.30 & 5.33 & 29 & 31 & 2.33 & 4.56 & 4.59 & 48 & 52 \\
& GL-Uni & 3.07 & 6.18 & 6.21 & 29 & 31 & 2.65 & 4.79 & 4.81 & 48 & 51 \\
\hline
\end{tabular}

The model is $y_{t}=1.4 \varrho^{0} \delta^{0} \mathbf{1}_{\left\{t>\left\lfloor T \lambda_{0}\right\rfloor\right\}}+\varrho^{0} y_{t-1}+e_{t}, e_{t} \sim$ i.i.d. $\mathscr{N}(0,0.5), \varrho^{0}=0.6, T=100$. The notes of Table 1 apply. 
Table 4: Small-sample coverage rates and lengths of the confidence sets for model M1

\begin{tabular}{|c|c|c|c|c|c|c|c|c|c|}
\hline \multirow{3}{*}{$\lambda_{0}=0.5$} & \multirow{4}{*}{$\begin{array}{c}\text { OLS-CR } \\
\text { Bai (1997) }\end{array}$} & \multicolumn{2}{|c|}{$\delta^{0}=0.4$} & \multicolumn{2}{|c|}{$\delta^{0}=0.8$} & \multicolumn{2}{|c|}{$\delta^{0}=1.2$} & \multicolumn{2}{|c|}{$\delta^{0}=1.6$} \\
\hline & & \multirow{2}{*}{$\frac{\text { Cov. }}{0.959}$} & \multirow{2}{*}{$\frac{\text { Lgth. }}{80.98}$} & \multirow{2}{*}{$\frac{\text { Cov. }}{0.958}$} & \multirow{2}{*}{$\frac{\text { Lgth. }}{62.61}$} & \multirow{2}{*}{$\frac{\text { Cov. }}{0.958}$} & \multirow{2}{*}{$\frac{\text { Lgth. }}{33.03}$} & \multirow{2}{*}{$\frac{\text { Cov. }}{0.937}$} & \multirow{2}{*}{$\frac{\text { Lgth. }}{15.60}$} \\
\hline & & & & & & & & & \\
\hline & & 0.803 & 60.84 & 0.837 & 32.03 & 0.874 & 15.84 & 0.875 & 9.34 \\
\hline & $\widehat{U}_{T}\left(T_{\mathrm{m}}\right) . n e q$ & 0.945 & 80.55 & 0.945 & 53.41 & 0.945 & 31.68 & 0.945 & 22.05 \\
\hline & ILR & 0.962 & 77.99 & 0.970 & 39.64 & 0.977 & 18.00 & 0.985 & 10.41 \\
\hline & GL-CR & 0.924 & 61.86 & 0.919 & 53.51 & 0.904 & 25.99 & 0.877 & 18.82 \\
\hline & GL-CR-Iter & 0.957 & 81.87 & 0.958 & 64.07 & 0.957 & 33.04 & 0.938 & 15.64 \\
\hline & sup-W & \multicolumn{2}{|c|}{0.300} & \multicolumn{2}{|c|}{0.865} & \multicolumn{2}{|c|}{0.996} & \multicolumn{2}{|c|}{0.997} \\
\hline \multirow[t]{7}{*}{$\lambda_{0}=0.35$} & OLS-CR & 0.960 & 79.26 & 0.920 & 61.18 & 0.956 & 32.07 & 0.938 & 15.58 \\
\hline & Bai (1997) & 0.828 & 59.47 & 0.833 & 43.92 & 0.871 & 15.96 & 0.877 & 9.39 \\
\hline & $\widehat{U}_{T}\left(T_{\mathrm{m}}\right)$. neq & 0.949 & 81.58 & 0.940 & 68.51 & 0.949 & 33.23 & 0.949 & 22.53 \\
\hline & ILR & 0.963 & 79.92 & 0.972 & 44.71 & 0.978 & 19.81 & 0.980 & 11.01 \\
\hline & GL-CR & 0.929 & 61.18 & 0.918 & 50.61 & 0.900 & 32.84 & 0.871 & 17.96 \\
\hline & GL-CR-Iter & 0.965 & 80.58 & 0.927 & 63.34 & 0.957 & 32.64 & 0.939 & 15.65 \\
\hline & sup-W & \multicolumn{2}{|c|}{0.276} & \multicolumn{2}{|c|}{0.592} & \multicolumn{2}{|c|}{0.992} & \multicolumn{2}{|c|}{1.000} \\
\hline \multirow[t]{7}{*}{$\lambda_{0}=0.2$} & OLS-CR & 0.954 & 78.14 & 0.967 & 54.93 & 0.972 & 29.74 & 0.951 & 15.73 \\
\hline & Bai (1997) & 0.827 & 59.48 & 0.884 & 33.24 & 0.900 & 17.11 & 0.896 & 9.84 \\
\hline & $\widehat{U}_{T}\left(T_{\mathrm{m}}\right) . n e q$ & 0.951 & 84.04 & 0.951 & 65.09 & 0.951 & 57.96 & 0.951 & 28.16 \\
\hline & ILR & 0.958 & 83.06 & 0.968 & 53.62 & 0.978 & 25.73 & 0.986 & 12.69 \\
\hline & GL-CR & 0.914 & 60.21 & 0.939 & 47.40 & 0.931 & 28.07 & 0.910 & 14.47 \\
\hline & GL-CR-Iter & 0.965 & 79.51 & 0.972 & 57.07 & 0.974 & 30.64 & 0.957 & 15.92 \\
\hline & sup-W & \multicolumn{2}{|c|}{0.161} & \multicolumn{2}{|c|}{0.576} & \multicolumn{2}{|c|}{0.901} & \multicolumn{2}{|c|}{0.987} \\
\hline
\end{tabular}

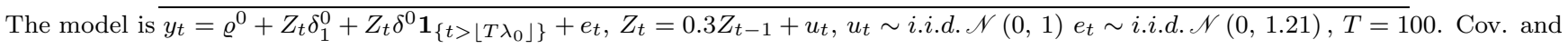
Lgth. refer to the coverage probability and the average length of the confidence set (i.e., the average number of dates in the confidence set). sup-W refers to the rejection probability of the sup-Wald test using a $5 \%$ asymptotic critical value. The number of simulations is 5,000 . 
Table 5: Small-sample coverage rates and lengths of the confidence sets for model M2

\begin{tabular}{|c|c|c|c|c|c|c|c|c|c|}
\hline \multirow{3}{*}{$\lambda_{0}=0.5$} & \multirow{4}{*}{$\begin{array}{c}\text { OLS-CR } \\
\text { Bai }(1997)\end{array}$} & \multicolumn{2}{|c|}{$\delta^{0}=0.4$} & \multicolumn{2}{|c|}{$\delta^{0}=0.8$} & \multicolumn{2}{|c|}{$\delta^{0}=1.2$} & \multicolumn{2}{|c|}{$\delta^{0}=1.6$} \\
\hline & & \multirow{2}{*}{$\frac{\text { Cov. }}{0.910}$} & \multirow{2}{*}{$\frac{\text { Lgth. }}{67.57}$} & \multirow{2}{*}{$\frac{\text { Cov. }}{0.911}$} & \multirow{2}{*}{$\frac{\text { Lgth. }}{68.87}$} & \multirow{2}{*}{$\frac{\text { Cov. }}{0.925}$} & \multirow{2}{*}{$\frac{\text { Lgth. }}{56.25}$} & \multirow{2}{*}{$\frac{\text { Cov. }}{0.945}$} & \multirow{2}{*}{$\frac{\text { Lgth. }}{42.30}$} \\
\hline & & & & & & & & & \\
\hline & & 0.808 & 67.57 & 0.811 & 50.22 & 0.843 & 32.67 & 0.894 & 20.74 \\
\hline & $\widehat{U}_{T}\left(T_{\mathrm{m}}\right)$.neq & 0.981 & 91.66 & 0.973 & 87.38 & 0.973 & 82.24 & 0.973 & 79.19 \\
\hline & ILR & 0.944 & 83.70 & 0.946 & 67.01 & 0.964 & 45.75 & 0.980 & 28.30 \\
\hline & GL-CR & 0.885 & 60.05 & 0.884 & 52.63 & 0.898 & 43.41 & 0.926 & 32.61 \\
\hline & GL-CR-Iter & 0.911 & 76.72 & 0.911 & 69.06 & 0.923 & 56.31 & 0.944 & 42.20 \\
\hline & sup-W & \multicolumn{2}{|c|}{0.333} & \multicolumn{2}{|c|}{0.565} & \multicolumn{2}{|c|}{0.878} & \multicolumn{2}{|c|}{0.926} \\
\hline \multirow[t]{7}{*}{$\lambda_{0}=0.35$} & OLS-CR & 0.927 & 75.58 & 0.910 & 66.20 & 0.921 & 52.89 & 0.944 & 39.15 \\
\hline & Bai (1997) & 0.838 & 66.86 & 0.821 & 49.34 & 0.857 & 32.43 & 0.893 & 20.77 \\
\hline & $\widehat{U}_{T}\left(T_{\mathrm{m}}\right)$. neq & 0.976 & 91.72 & 0.976 & 87.25 & 0.976 & 81.76 & 0.976 & 77.62 \\
\hline & ILR & 0.941 & 83.24 & 0.945 & 68.31 & 0.959 & 47.58 & 0.975 & 29.32 \\
\hline & GL-CR & 0.898 & 57.32 & 0.888 & 50.29 & 0.902 & 43.08 & 0.924 & 29.06 \\
\hline & GL-CR-Iter & 0.930 & 75.87 & 0.913 & 66.13 & 0.921 & 52.66 & 0.944 & 38.71 \\
\hline & sup-W & \multicolumn{2}{|c|}{0.489} & \multicolumn{2}{|c|}{0.625} & \multicolumn{2}{|c|}{0.868} & \multicolumn{2}{|c|}{1.000} \\
\hline \multirow[t]{7}{*}{$\lambda_{0}=0.2$} & OLS-CR & 0.910 & 75.24 & 0.917 & 64.17 & 0.931 & 48.76 & 0.953 & 34.26 \\
\hline & Bai (1997) & 0.808 & 67.03 & 0.852 & 50.40 & 0.897 & 33.62 & 0.937 & 21.76 \\
\hline & $\widehat{U}_{T}\left(T_{\mathrm{m}}\right) \cdot$.neq & 0.981 & 92.17 & 0.981 & 89.14 & 0.981 & 84.72 & 0.981 & 80.39 \\
\hline & ILR & 0.938 & 85.37 & 0.951 & 74.32 & 0.963 & 57.03 & 0.977 & 36.24 \\
\hline & GL-CR & 0.912 & 56.87 & 0.909 & 48.68 & 0.920 & 38.47 & 0.932 & 23.91 \\
\hline & GL-CR-Iter & 0.894 & 75.15 & 0.923 & 64.14 & 0.934 & 48.75 & 0.953 & 34.06 \\
\hline & sup-W & \multicolumn{2}{|c|}{0.331} & \multicolumn{2}{|c|}{0.565} & \multicolumn{2}{|c|}{0.795} & \multicolumn{2}{|c|}{0.931} \\
\hline
\end{tabular}

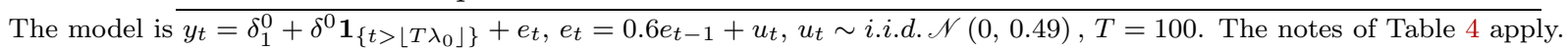


Table 6: Small-sample coverage rates and lengths of the confidence sets for model M3

\begin{tabular}{|c|c|c|c|c|c|c|c|c|c|}
\hline & & \multicolumn{2}{|c|}{$\delta^{0}=0.4$} & \multicolumn{2}{|c|}{$\delta^{0}=0.8$} & \multicolumn{2}{|c|}{$\delta^{0}=1.2$} & \multicolumn{2}{|c|}{$\delta^{0}=1.6$} \\
\hline & & Cov. & Lgth. & Cov. & Lgth. & Cov. & Lgth. & Cov. & Lgth. \\
\hline \multirow[t]{7}{*}{$\lambda_{0}=0.5$} & OLS-CR & 0.895 & 67.38 & 0.923 & 39.66 & 0.952 & 19.23 & 0.972 & 10.30 \\
\hline & Bai (1997) & 0.759 & 43.84 & 0.851 & 17.50 & 0.904 & 8.77 & 0.935 & 5.49 \\
\hline & $\widehat{U}_{T}\left(T_{\mathrm{m}}\right)$. neq & 0.948 & 81.26 & 0.947 & 71.72 & 0.949 & 80.79 & 0.952 & 90.23 \\
\hline & ILR & 0.934 & 77.87 & 0.951 & 50.62 & 0.961 & 31.01 & 0.975 & 22.80 \\
\hline & GL-CR & 0.871 & 66.58 & 0.876 & 36.80 & 0.893 & 15.76 & 0.914 & 7.50 \\
\hline & GL-CR-Iter & 0.896 & 71.50 & 0.916 & 42.49 & 0.948 & 20.23 & 0.967 & 10.30 \\
\hline & sup-W & \multicolumn{2}{|c|}{0.300} & \multicolumn{2}{|c|}{0.988} & \multicolumn{2}{|c|}{0.996} & \multicolumn{2}{|c|}{1.000} \\
\hline \multirow[t]{7}{*}{$\lambda_{0}=0.35$} & OLS-CR & 0.902 & 66.58 & 0.938 & 39.84 & 0.953 & 19.71 & 0.973 & 10.47 \\
\hline & Bai (1997) & 0.766 & 43.21 & 0.855 & 17.61 & 0.901 & 8.82 & 0.935 & 5.52 \\
\hline & $\widehat{U}_{T}\left(T_{\mathrm{m}}\right)$. neq & 0.950 & 82.13 & 0.950 & 72.22 & 0.952 & 78.81 & 0.953 & 87.02 \\
\hline & ILR & 0.936 & 78.13 & 0.944 & 47.77 & 0.959 & 30.81 & 0.976 & 22.16 \\
\hline & GL-CR & 0.885 & 66.06 & 0.887 & 35.98 & 0.893 & 15.79 & 0.917 & 7.59 \\
\hline & GL-CR-Iter & 0.908 & 71.08 & 0.937 & 42.99 & 0.951 & 20.71 & 0.967 & 10.55 \\
\hline & sup-W & \multicolumn{2}{|c|}{0.595} & \multicolumn{2}{|c|}{0.973} & \multicolumn{2}{|c|}{1.000} & \multicolumn{2}{|c|}{1.000} \\
\hline \multirow[t]{7}{*}{$\lambda_{0}=0.2$} & OLS-CR & 0.910 & 65.50 & 0.950 & 38.42 & 0.956 & 18.13 & 0.969 & 10.41 \\
\hline & Bai (1997) & 0.802 & 44.43 & 0.884 & 18.47 & 0.952 & 8.68 & 0.932 & 5.32 \\
\hline & $\widehat{U}_{T}\left(T_{\mathrm{m}}\right)$. neq & 0.948 & 89.91 & 0.949 & 77.46 & 0.950 & 79.01 & 0.932 & 88.41 \\
\hline & ILR & 0.932 & 79.97 & 0.941 & 49.72 & 0.965 & 33.97 & 0.951 & 21.62 \\
\hline & GL-CR & 0.897 & 57.65 & 0.903 & 34.51 & 0.889 & 15.21 & 0.912 & 7.43 \\
\hline & GL-CR-Iter & 0.903 & 65.07 & 0.923 & 37.65 & 0.939 & 17.46 & 0.959 & 10.39 \\
\hline & sup-W & \multicolumn{2}{|c|}{0.443} & \multicolumn{2}{|c|}{0.914} & \multicolumn{2}{|c|}{0.999} & \multicolumn{2}{|c|}{1.000} \\
\hline
\end{tabular}

